\title{
Iterated monodromy groups of quadratic polynomials, I
}

\author{
Laurent Bartholdi and Volodymyr V. Nekrashevych*
}

\begin{abstract}
We describe the iterated monodromy groups associated with post-critically finite quadratic polynomials, and make explicit their connection to the 'kneading sequence' of the polynomial.

We then give recursive presentations by generators and relations for these groups, and study some of their properties, like torsion and 'branchness'.
\end{abstract}

Mathematics Subject Classification (2000). 37F10, 20E08, 37F20.

Keywords. Iterated monodromy groups, quadratic polynomials, automata groups, kneading sequences, recursive presentations.

\section{Introduction}

Symbolic dynamics of quadratic complex polynomials are traditionally studied by their "kneading sequence", an infinite sequence over the alphabet $\{0,1, *\}$ that encodes symbolically the dynamics of the map (see [9], [16]). This sequence is (pre)periodic if the polynomial has finite post-critical set, and we shall make that assumption here.

A construction by the second author associates a finitely generated group, defined by automata, with such a polynomial. This group is the iterated monodromy group of the polynomial, and acts on a binary rooted tree.

In this paper, we show that the automata defining the group may be chosen in a particularly simple manner. These automata are "bounded", i.e., their activity is trivial away from a ray in the tree. If the tree's rays are labeled by infinite $\{0,1\}$ sequences, then the rays on which activity is non-trivial are the translates of the periodic part of the kneading sequence of the polynomial.

Actually, we study a class of groups containing all the iterated monodromy groups of post-critically finite quadratic polynomials as a proper subset. The first set of such groups $\Omega(v)$ corresponds to kneading sequences of the form $(v *)^{\omega}$, and the second set of groups $\Re(w, v)$ corresponds to kneading sequences of the form $w\left(v^{\omega}\right)$.

*The second author acknowledges partial support by NSF grant DMS-0605019. 
Since not every sequence of this form is realizable as a kneading sequence, not all groups $\Omega(v)$ and $\Omega(w, v)$ are iterated monodromy groups of quadratic polynomials. In particular, if $v$ is periodic (is a proper power of a word), then $\Omega(w, v)$ is not an iterated monodromy group of any polynomial (they are iterated monodromy groups of obstructed topological polynomials). In all the other cases the corresponding groups are the iterated monodromy groups of some post-critically finite polynomials of degree $2^{n}$. For more details see a general description of the iterated monodromy groups of post-critically finite polynomials in [17].

We describe elementary properties of the groups $\Omega(v)$ and $\Omega(w, v)$. Unsurprisingly, the groups are of a very different nature depending on whether the kneading sequence is periodic (for $\Re(v)$ ) or pre-periodic (for $\Re(w, v)$ ):

- if the sequence is periodic, these groups are weakly branch, but not branch; they are torsion-free;

- if the sequence is pre-periodic, these groups are branch, and contain elements of arbitrarily high 2-power order, as well as elements of infinite order.

We give in all cases recursive presentations for these groups.

The paper is organised as follows: Section 2 contains necessary recollections on iterated monodromy groups; Section 3 describes the groups $\mathscr{R}(v)$ associated with periodic kneading sequences; Section 4 describes the groups $\Omega(w, v)$ associated with pre-periodic kneading sequences; and Section 5 proves that these groups are indeed the iterated monodromy groups of polynomials with prescribed kneading sequence.

Our aim in this paper was to show that kneading sequences and bounded automata are really equivalent descriptions for post-critically finite quadratic polynomials and to describe the basic algebraic properties of the iterated monodromy groups.

Some of the groups we consider already appeared in the literature, and the present paper extends or unifies algebraic results concerning them. The groups $\mathscr{\Omega}(v)$ and $\Omega(w, v)$ that coincide with previously known ones are

- $\Omega()=\mathbb{Z}$;

- $\Omega(0,1)=\Re(1,0)=$ the infinite dihedral group;

- $\Omega(0)$, an "amenable non-subexponentially-amenable" group considered in [7], where it is called the "Basilica group";

- $\Omega(0,11)$, a group whose growth is studied in [12];

- $\Omega(0,111)$, a group whose Lie algebra is studied in [2], and whose presentation and growth are studied in [3], where it is called the "Grigorchuk overgroup". This group contains Grigorchuk's example of an infinite torsion group [13] as a subgroup. 
1.1. Notation. The notation we use is quite standard, with groups acting on the right. We therefore write $[a, b]=a^{-1} b^{-1} a b$, and $a^{b}=b^{-1} a b$. More generally, we write $g^{k_{1} h_{1}+\cdots+k_{n} h_{n}}=\left(g^{k_{1}}\right)^{h_{1}} \ldots\left(g^{k_{n}}\right)^{h_{n}}$ for $k_{i} \in \mathbb{Z}$ and $g, h_{i}$ group elements. The derived subgroup $[G, G]$ of $G$ is written $G^{\prime}$. The cyclic group of order $n$ is written $\frac{\mathbb{Z}}{n \mathbb{Z}}$.

The notation $a=\left\langle\left\langle a_{0}, a_{1}\right\rangle\right\rangle \sigma$ describes an automorphism $a$ of the binary tree. It first acts as $a_{0}$ on the left subtree and as $a_{1}$ on the right subtree, and then applies the permutation $\sigma$ to these two subtrees.

On the other hand, if $g$ is an element of a free group $F$ that acts (in principle non-faithfully) on a tree, this action will in our cases be described by a map $\Psi: F \rightarrow$ $(F \times F) \rtimes \mathfrak{S}_{2}$. Then the notation $\Psi(g)=\left\langle\left\langle g_{0}, g_{1}\right\rangle\right\rangle \sigma$ describes the image of $g$ using the same convention as above.

If $v$ is a finite word over an alphabet $X$ then $|v|$ denotes its length, i.e., such $n$ that $v \in X^{n}$.

\section{Iterated monodromy and self-similar groups}

2.1. Iterated monodromy groups. We present in this subsection a review of the notions and results related to iterated monodromy groups. More details can be found in [17].

A polynomial $f \in \mathbb{C}[z]$ is said to be post-critically finite if the orbit of every critical point is finite. The union $P_{f}=\bigcup_{n \geq 1} f^{\circ n}\left(C_{f}\right)$ of the orbits of critical points is called the post-critical set of $f$; here $C_{f}$ is the set of critical points of $f$.

Suppose that $f$ is a post-critically finite polynomial. We consider it as a branched self-covering of the complex plane. Moreover, it is a covering map $f: \mathbb{C} \backslash f^{-1}\left(P_{f}\right) \rightarrow$ $\mathbb{C} \backslash P_{f}$ of the punctured plane $\mathbb{C} \backslash P_{f}$ by its open subset $\mathbb{C} \backslash f^{-1}\left(P_{f}\right)$.

Consider now the general situation of a covering $f: \mathcal{M}_{1} \rightarrow \mathcal{M}$ of a path connected and locally path connected topological space $\mathcal{M}$ by an open subset $\mathcal{M}_{1}$. We can iterate the partially defined map $f$ to obtain coverings $f^{n}: \mathcal{M}_{n} \rightarrow \mathcal{M}$ of $\mathcal{M}$ by open subsets.

Take a basepoint $t \in \mathcal{M}$. Then the fundamental group $\pi_{1}(\mathcal{M}, t)$ acts naturally on the set of preimages $f^{-n}(t)$ : the image of a point $z \in f^{-n}(t)$ under the action of a loop $\gamma \in \pi_{1}(\mathcal{M}, t)$ is equal to the endpoint of the $f^{n}$-preimage of $\gamma$ that starts at $z$. Such a preimage exists and is unique, since $f^{n}: \mathcal{M}_{n} \rightarrow \mathcal{M}$ is a covering.

In this way, we get an action of $\pi_{1}(\mathcal{M}, t)$ on the formal disjoint union $\bigsqcup_{n \geq 0} f^{-n}(t)$, the backward orbit of $t$. The quotient of $\pi_{1}(\mathcal{M}, t)$ by the kernel of its action is called the iterated monodromy group of $f$ and is denoted $\operatorname{IMG}(f)$.

The backward orbit $T$ has a natural structure of a rooted tree. The root is the unique element $t$ of the set $f^{-0}(t)$ and the vertex $z \in f^{-n}(t)$ of the $n$th level of the tree is connected to the vertex $f(z) \in f^{-(n-1)}(t)$ of the $(n-1)$-st level.

It is easy to see that the actions of the fundamental group and of the iterated monodromy group preserve the structure of the rooted tree. 
Suppose that the degree of the covering $f$ (i.e., the number of preimages of a point) is finite and is equal to $d$. Choose an alphabet $X$ of $d$ letters and consider the tree of words $X^{*}$ over this alphabet, i.e., the free monoid generated by $X$, in which a word $v$ is connected to all words of the form $v x$ for $x \in X$. The root of $X^{*}$ is the empty word. Since both the tree of words $X^{*}$ and the tree of preimages $T$ are regular $d$-trees, they are isomorphic.

A particularly nice class of isomorphisms $\Lambda: X^{*} \rightarrow T$ are constructed in the following way. Choose an arbitrary bijection $\Lambda: X \rightarrow f^{-1}(t)$ of the first level of the tree $X^{*}$ with the first level of the tree $T$. Choose also a collection of connecting paths $\ell_{x}$ from $t$ to $\Lambda(x)$ in $\mathcal{M}$.

We define now the isomorphism $\Lambda$ inductively level by level. It is already defined on the zeroth and first levels. Suppose that it is defined on the $n$th level. Let $v \in X^{n}$ be an arbitrary vertex of the $n$th level and let $x \in X$ be an arbitrary letter. Then the path $\ell_{x}$ has a unique $f^{n}$-preimage starting at $\Lambda(v)$. We declare $\Lambda(x v)$ to be the endpoint of this preimage.

The map $\Lambda: X^{*} \rightarrow T$ that we just constructed is an isomorphism of rooted trees, and the following proposition makes it possible to compute the action of elements of the fundamental group on the tree $T$.

Proposition 2.1. Let us conjugate the action of $\pi_{1}(\mathcal{M}, t)$ on the tree $T$ by the isomorphism $\Lambda: X^{*} \rightarrow T$ constructed above. Then the resulting action of $\pi_{1}(\mathcal{M}, t)$ on $X^{*}$ is computed by the following recursive formula:

$$
(x v)^{\gamma}=y(v)^{\ell_{x} \gamma_{x} \ell_{y}^{-1}}
$$

where $x \in X, v \in X^{*}$ and $\gamma \in \pi_{1}(\mathcal{M}, t)$ are arbitrary, $\gamma_{x}$ is the $f$-preimage of $\gamma$ starting at $\Lambda(x)$ and $y$ is such that $\Lambda(y)$ is the end of $\gamma_{x}$.

2.2. Automata. Recursive formulæ as in Proposition 2.1 are conveniently interpreted in terms of automata theory. We interpret $\operatorname{IMG}(f)\left(\right.$ or $\left.\pi_{1}(\mathcal{M}, t)\right)$ as an automaton which being in a state $\gamma$ and reading as input a letter $x$ produces as output the letter $y$ and moves to state $\ell_{x} \gamma_{x} \ell_{y}^{-1}$. Then it is ready to read a new input.

We describe such automata (or their subsets) by their Moore diagram. It is a graph whose vertices are identified with the states of the automaton, in which we have an arrow from a state $q_{1}$ to a state $q_{2}$ labeled by a letter $x \in X$ if the automaton, being in state $q_{1}$, after reading the letter $x$ goes to the state $q_{2}$. We also label the vertices of the automaton by the transformations of the letters that they define.

In particular, if $X=\{0,1\}$ and the automaton describes an action of a group $G$ on the tree $X^{*}$, then every state $g \in G$ acts either by the rule $(0 w)^{g}=0(w)^{g_{0}}$ and $(1 w)^{g}=1(w)^{g_{1}}$ or by the rule $(0 w)^{g}=1(w)^{g_{0}}$ and $(1 w)^{g}=0(w)^{g_{1}}$, where $g_{0}$, $g_{1}$ are next states of the automaton and $w \in X^{*}$ is arbitrary. In the first case we say that $g$ is inactive and write $g=\left\langle\left\langle g_{0}, g_{1}\right\rangle\right\rangle$, in the second case we say that $g$ is active 
and write $g=\left\langle\left\langle g_{0}, g_{1}\right\rangle\right\rangle \sigma$. In both cases we have in the Moore diagram an arrow from $g$ to $g_{i}$ labeled by $i$. We will mark the inactive states in a Moore diagram by white dots, and the active states by black dots.

The notations $g=\left\langle\left\langle g_{0}, g_{1}\right\rangle\right\rangle$ and $g=\left\langle\left\langle g_{0}, g_{1}\right\rangle\right\rangle \sigma$ come from the decomposition of the automorphism group aut $\left(X^{*}\right)$ of the binary rooted tree into the (permutational) wreath product aut $\left(X^{*}\right) \succ \widetilde{\Xi}_{2}=\operatorname{aut}\left(X^{*}\right)^{X} \rtimes \Im_{2}$. The factor $\Im_{2}$ acts on the tree just by its action on the first letter of words, and the factor aut $\left(X^{*}\right)^{X}$ acts separately on the each of the subtrees $x X^{*}$ :

$$
(0 v)^{\left\langle\left\langle g_{0}, g_{1}\right\rangle\right\rangle}=0\left(v^{g_{0}}\right), \quad(1 v)^{\left\langle\left\langle g_{0}, g_{1}\right\rangle\right\rangle}=1\left(v^{g_{1}}\right) .
$$

Then $\sigma \in \widetilde{S}_{2}$ denotes the transposition and $\left\langle\left\langle g_{0}, g_{1}\right\rangle\right\rangle \sigma$ is equal to the composition of $\left\langle\left\langle g_{0}, g_{1}\right\rangle\right\rangle$ and $\sigma$.

\subsection{Self-similar groups}

Definition 1. A group $G$ acting faithfully on the tree $X^{*}$ is said to be self-similar if for every $g \in G$ and every $x \in X$ there exist $h \in G$ and $y \in X$ such that

$$
(x w)^{g}=y w^{h}
$$

for all $w \in X^{*}$.

In other terms a self-similar group is an automaton such that the set of transformations of $X^{*}$ defined by its states is a group with respect to composition.

In particular, Proposition 2.1 shows that iterated monodromy groups act on $X^{*}$ self-similarly (i.e., they are self-similar groups).

If $G$ is a self-similar group acting on the binary tree then each of its elements $g$ is decomposed either as $g=\left\langle\left\langle g_{0}, g_{1}\right\rangle\right\rangle$ or as $g=\left\langle\left\langle g_{0}, g_{1}\right\rangle\right\rangle \sigma$ according to its action on $X \subset X^{*}$ and, by the definition of self-similarity, we have $g_{0}, g_{1} \in G$.

Hence every self-similar group $G$ comes with an associated wreath recursion $G \rightarrow G>\subseteq_{X}$. On the other hand, every such homomorphism defines recursively an action of $G$ on $X^{*}$ (which is also called self-similar, though it may be non-faithful).

If $\Psi: G \rightarrow G \imath \widetilde{S}_{X}$ is a homomorphism, then for $g \in G$ and $v \in X^{*}$ we define the restriction $\left.g\right|_{v}$ recursively by the rule

$$
g=\left\langle\left\langle\left. g\right|_{0},\left.g\right|_{1}\right\rangle\right\rangle \pi, \quad \pi \in \widetilde{S}_{X}
$$

and

$$
\left.g\right|_{v x}=\left.\left.g\right|_{v}\right|_{x}
$$

for all $v \in X^{*}$ and $x \in X$. We also assume that $\left.g\right|_{\emptyset}=g$, where $\emptyset$ denotes the empty word. 
If the action of $G$ on $X^{*}$ is faithful, then the restrictions are uniquely defined by the condition

$$
(v u)^{g}=v^{g} u^{g \mid v}
$$

for $v, u \in X^{*}$.

Proposition 2.2. Let $G$ be a self-similar group and let $N$ be a normal subgroup of $G$ which belongs to the stabilizer of the first level. If $G / N$ is torsion free, then so is $G$.

Proof. Suppose that $g \in G$ has finite order. Since $G / N$ is torsion free, we have $g \in N$. This implies that $g$ belongs to the stabilizer of the first level, so $\left.\left(g^{n}\right)\right|_{x}=$ $\left(\left.g\right|_{x}\right)^{n}$ for all $x \in X$. The elements $\left.g\right|_{x}$ therefore also have finite order. Repeating the same argument, we obtain inductively that $g$ fixes $X^{*}$, so $g=1$.

Definition 2. A self-similar group $G$ is called recurrent (or self-replicating) if its action is transitive on the first level of the tree $X^{*}$ and if for some (and thus for all) $x \in X$ the homomorphism $\left.g \mapsto g\right|_{x}: G_{x} \rightarrow G$ is onto, where $G_{x}$ is the stabilizer of $x$ in $G$.

It is easy to prove that if a self-similar group is recurrent, then it is transitive on every level of the tree $X^{*}$ (the group is then called level-transitive).

Definition 3. A homomorphism $\Psi: G \rightarrow G \curlywedge \Im_{X}$ is contracting if there exits a finite set $N \subset G$ such that for every $g \in G$ there exists $n \in \mathbb{N}$ such that $\left.g\right|_{v} \in N$ for all words $v$ of length $\geq n$. The smallest such set $N$ is called the nucleus of $\Psi$.

We say that a self-similar group is contracting if the associated wreath recursion is contracting. The nucleus of the recursion is called then the nucleus of the group.

It follows directly from the definitions that the nucleus is an automaton, i.e., that for every element $g$ of the nucleus and for every $x \in X$ the restriction $\left.g\right|_{x}$ is in the nucleus.

\subsection{Bounded automata}

Definition 4. An automorphism $g \in \operatorname{aut}\left(X^{*}\right)$ is finite state if it is defined by a finite automaton, or, equivalently, if the set $\left\{\left.g\right|_{v} \mid v \in X^{*}\right\}$ is finite.

An automorphism $g \in \operatorname{aut}\left(X^{*}\right)$ is called bounded if it is finite-state and the sequence

$$
q_{n}=\left|\left\{v \in X^{n}|g|_{v} \neq 1\right\}\right|
$$

is bounded. Here 1 is the identity tree automorphism. 
It is not hard to see that the set of all bounded automorphisms of the tree $X^{*}$ is a group.

Bounded automata and tree automorphisms were defined and studied for the first time by S. Sidki in [18]. The following description of bounded automata follows from his results.

Definition 5. An automorphism $g \in \operatorname{aut}\left(X^{*}\right)$ is called finitary if there exists $n \in \mathbb{N}$ such that $\left.g\right|_{v}=1$ for all $v \in X^{*}$ of length more than $n$.

An automorphism $g \in \operatorname{aut}\left(X^{*}\right)$ is called directed if there exists $v \in X^{*}$ such that $\left.g\right|_{v}=g$ and $\left.g\right|_{u}$ is finitary for every $u \in X^{*}$ such that $u \neq v$ and $|u|=|v|$. Then the infinite word $v^{\omega}=v v v \ldots$ is called the kneading sequence of $g$.

If $g$ is finitary, then it acts only on the first $n$ letters of every word for some fixed $n$. If it is directed, then all of its non-trivial action on the tree is concentrated around the path described by the kneading sequence.

It is easy to see that every finitary and every directed automorphism is bounded.

Proposition 2.3. If $g \in \operatorname{aut}\left(X^{*}\right)$ is bounded, then there exists $n$ such that $\left.g\right|_{v}$ for every $v \in X^{n}$ is either finitary or directed.

We have the following properties of groups of bounded automata.

Theorem 2.4 ([8]). If $G$ acts on $X^{*}$ by bounded automata, is finitely generated and self-similar, then it is contracting.

Theorem 2.5 ([5]). The group of all bounded automorphisms of the tree $X^{*}$ is amenable.

2.5. Branch groups. We say that a group $G$ acting on a regular tree $X^{*}$ is regular weakly branch on $H \leq G$ if $H$ is non-trivial and contains the geometric direct product $H \times \cdots \times H$ with $|X|$ factors. Here by geometric direct product we mean the group generated by copies of $H$ acting disjointly on all subtrees $x X^{*}$ with $x$ ranging over $X$. The group $G$ is regular branch on $H$ if it is weakly branch on some subgroup $H$ that has finite index in $G$.

Proposition 2.6 ([4], Theorem 6.9). Let $G$ be a regular weakly branch group on its subgroup $L$, and suppose that a subgroup $K$ of $L$ has a regular orbit on $X^{n}$ for some n. Then $G$ contains $\lim \imath^{n} K=\bigcup_{n \geq 0} K \imath \cdots>K$.

In particular, if there exists such $a K \cong \frac{\mathbb{Z}}{p \mathbb{Z}}$, then $G$ contains every finite p-group.

Proof. Since $G$ is weakly branch, it contains the subgroup $K_{0}=K$, and for all $i \geq 1$ the subgroup $K_{i}=1 \times \cdots \times K_{i-1} \times \cdots \times 1$ with $|X|^{n}$ factors, where the $K_{i-1}$ is at any position on a regular orbit of $K$. 
The group generated by $K_{0} \cup K_{1} \cup \cdots$ is isomorphic to $\lim 2^{n} K$.

It is known [15] that every extension embeds in a wreath product; since finite $p$-groups are iterated extensions of $\frac{\mathbb{Z}}{p \mathbb{Z}}$, the second claim follows.

\section{The groups $\Re(v)$}

Let $v=x_{1} x_{2} \ldots x_{n-1}$ be a word over the alphabet $X=\{0,1\}$. We denote by $\Re(v)$ the subgroup of aut $\left(X^{*}\right)$ generated by the elements $a_{1}, \ldots, a_{n}$ defined by

$$
a_{1}=\left\langle\left\langle 1, a_{n}\right\rangle\right\rangle \sigma, \quad a_{i+1}= \begin{cases}\left\langle\left\langle a_{i}, 1\right\rangle\right\rangle & \text { if } x_{i}=0, \\ \left\langle\left\langle 1, a_{i}\right\rangle\right\rangle & \text { if } x_{i}=1,\end{cases}
$$

when $1 \leq i<n$.

In other words, the group $\Omega(v)$ is generated by the automaton whose Moore diagram is shown on Figure 1. In this diagram, only the edges leading to non-trivial states are drawn, and the active state $a_{1}$ is labelled by $\sigma$.

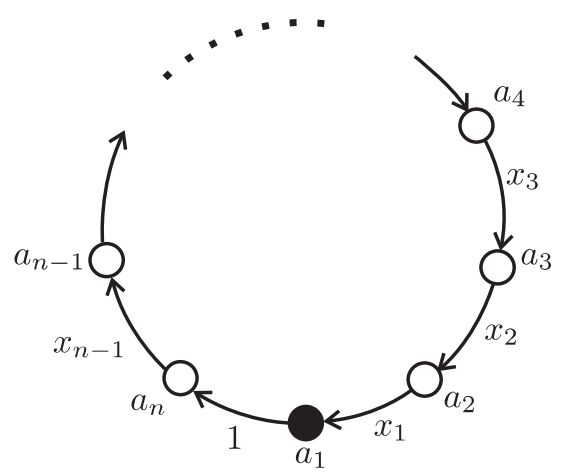

Figure 1. Automaton generating $\Re(v)$.

Note that, in the group $\Re(v)$, if we replace the generators $a_{i}$ by their inverses $a_{i}^{\prime}$, we obtain the description

$$
a_{1}^{\prime}=\left\langle\left\langle a_{n}^{\prime}, 1\right\rangle\right\rangle \sigma, \quad a_{i+1}^{\prime}= \begin{cases}\left\langle\left\langle a_{i}^{\prime}, 1\right\rangle\right\rangle & \text { if } x_{i}=0, \\ \left\langle\left\langle 1, a_{i}^{\prime}\right\rangle\right\rangle & \text { if } x_{i}=1,\end{cases}
$$

when $1 \leq i<n$. Therefore, if we modify the definition of $\Re(v)$ by setting $a_{1}=$ $\left\langle\left\langle a_{n}, 1\right\rangle\right\rangle \sigma$, we do not change the group.

Let us denote by $v^{\prime}$ the word obtained from the word $v$ by changing 0 to 1 and 1 to 0 . It is easy to see then that the groups $\Omega(v)$ and $\Omega\left(v^{\prime}\right)$ are conjugate; more precisely, $\Omega\left(v^{\prime}\right)=\Omega(v)^{\alpha}$, where $\alpha \in \operatorname{aut}\left(X^{*}\right)$ is defined by the recursion $\alpha=\langle\langle\alpha, \alpha\rangle\rangle \sigma$. Note that $\alpha$ also interchanges 0 and 1, i.e., $v^{\alpha}=v^{\prime}$. 
Lemma 3.1. The group $\Omega(v)$ is recurrent and level-transitive.

Proof. The projections of the elements $a_{i+1}, a_{i+1}^{a_{1}}$ and $a_{1}^{2}$, all fixing the first level of $X^{*}$, give all generators; the action is therefore recurrent.

To check that the action of a recurrent group is level-transitive, it suffices to check that it is transitive on the first level; this is achieved by $a_{1}$.

3.1. Wreath recursion. Let us denote by $F_{n}$ the free group on $\left\{a_{1}, \ldots, a_{n}\right\}$, and by $\widetilde{S}_{2}=\{1, \sigma\}$ the symmetric group on $X=\{0,1\}$. Let $\Psi: F_{n} \rightarrow F_{n} \prec \widetilde{S}_{2}$ be given by the recursive definition of the group $\Re(v)$, i.e.,

$$
\Psi\left(a_{1}\right)=\left\langle\left\langle 1, a_{n}\right\rangle\right\rangle \sigma, \quad \Psi\left(a_{i+1}\right)= \begin{cases}\left\langle\left\langle a_{i}, 1\right\rangle\right\rangle & \text { if } x_{i}=0, \\ \left\langle\left\langle 1, a_{i}\right\rangle\right\rangle & \text { if } x_{i}=1,\end{cases}
$$

when $1 \leq i<n$.

We can iterate the map $\Psi$ to obtain a homomorphism $\left.\Psi^{k}: F_{n} \rightarrow F_{n} \imath \widetilde{S}_{2} \nmid \cdots\right\urcorner \widetilde{S}_{2}$, where $\widetilde{S}_{2}$ appears $k$ times in the iterated permutational wreath product.

Lemma 3.2. The wreath recursion $\Psi$ is contracting: if $\Psi^{n}(g)=\left\langle\left\langle g_{0}, \ldots, g_{2^{n}-1}\right\rangle\right\rangle \pi$ and $\|g\|>2$, then $\left\|g_{i}\right\|<\|g\|$ for all $i$.

The nucleus of $\Omega(v)$ (and of the wreath recursion $\Psi$ ) may be expressed as follows: let $d$ be maximal such that $v x=u^{d}$ for some $x \in\{0,1\}$. Then the nucleus of $\Omega(v)$ contains $1+n(d+1)$ elements. Taking indices modulo $n$, they are $1, a_{i}^{ \pm 1}$, and $a_{i}^{\epsilon} a_{i+(n / d) j}^{-\epsilon}$ for any $j \in\{1, \ldots, d-1\}$, with the sign $\epsilon=1$ if $x_{n / d}=x=0$ and $\epsilon=-1$ if $x_{n / d}=x=1$.

Proof. Let us prove that the described set of elements of $F_{n}$ is the nucleus of the recursion $\Psi$.

It follows directly from the wreath recursion that if $\Psi(g)=\left\langle\left\langle g_{0}, g_{1}\right\rangle\right\rangle \sigma^{t}$, then $\left\|g_{0}\right\|+\left\|g_{1}\right\| \leq\|g\|$. Let us call an element $g$ stable if $\left\|g_{i}\right\|=\|g\|$ for some $i \in\{0,1\}$ and the element $g_{i}$ is also stable. In other words, an element $g \in F_{k}$ is stable if for every $k$ there exists a word $v_{k} \in X^{k}$ (which is necessarily unique) such that $\left\|\left.g\right|_{v_{k}}\right\|=\|g\|$. Note then that for every $u \in X^{k}$ different from $v_{k}$ we have $\left.g\right|_{u}=1$, and $v_{k}$ is the prefix of $v_{k+1}$. Hence there exists an infinite sequence $w_{g}$ such that $v_{k}$ is the beginning of length $k$ of $w_{g}$. We call the sequence $w_{g}$ the kneading sequence of $g$.

If we prove that the set of stable elements is finite, we will prove that the recursion is contracting and the nucleus is the set of stable elements, since the length of elements is non-increasing under taking restrictions.

It is easy to see that if $g$ is stable, then $g^{-1}$ is also stable and $w_{g}-1=\left(w_{g}\right)^{g}$. If $g$ is stable and $g=a_{i_{1}}^{\epsilon_{1}} a_{i_{2}}^{\epsilon_{2}} \ldots a_{i_{k}}^{\epsilon_{k}}$ for $\epsilon_{i} \in\{ \pm 1\}$ and $k=|g|$, then $w_{g}$ is also the kneading sequence of $a_{i_{1}}^{\epsilon_{1}},\left(w_{g}\right)^{a_{i_{1}}^{\epsilon_{1}}}$ is the kneading sequence of $a_{i_{2}}^{\epsilon_{2}},\left(w_{g}\right)^{a_{i_{1}}^{\epsilon_{1}} a_{i_{2}}^{\epsilon_{2}}}$ 
is the kneading sequence of $a_{i_{3}}^{\epsilon_{3}}$, etc. Otherwise the length of $g$ would decrease along $w_{g}$. Hence the set of kneading sequences of the stable elements coincides with the set $K$ of kneading sequences of the generators and their inverses. Let $\Gamma$ be the graph with set of vertices $K$ in which for every $a_{i}$ there is an edge from the kneading sequence $w_{a_{i}}$ to the kneading sequence $w_{a_{i}^{-1}}=\left(w_{a_{i}}\right)^{a_{i}}$ labeled by $a_{i}$ (if we go in the opposite direction then the edge is labeled by $\left.a_{i}^{-1}\right)$. Then for every stable element $g=a_{i_{1}}^{\epsilon_{1}} a_{i_{2}}^{\epsilon_{2}} \ldots a_{i_{k}}^{\epsilon_{k}}$ there exists a path (without back-tracking) in the graph $\Gamma$ labeled by $a_{i_{1}}^{\epsilon_{1}}, a_{i_{2}}^{\epsilon_{2}}, \ldots, a_{i_{k}}^{\epsilon_{k}}$.

It follows from the definitions that the kneading sequence of the element $a_{i}$ is $\left(x_{i} x_{i-1} \ldots x_{1} 1 x_{n-1} \ldots x_{i+1}\right)^{\omega}$ and the kneading sequence of $a_{i}^{-1}$ is $\left(x_{i} x_{i-1} \ldots x_{1} 0 x_{n-1} \ldots x_{i+1}\right)^{\omega}$. Let, as above, $d$ be the maximal number such that $v x=u^{d}$ for some $x \in\{0,1\}$. We denote by $x^{\prime} \neq x$ the other letter of the alphabet. If $x=1$, then the kneading sequence of $a_{i}$ is of period $n / d$ and the kneading sequence of $a_{i}^{-1}$ is of period $n$. If $x=0$, then the period of the kneading sequence of $a_{i}$ is $n$ and the period of the kneading sequence of $a_{i}^{-1}$ is $n / d$. In each case the set $K$ of kneading sequences of stable elements of $\Re(v)$ contains $n+n / d$ elements. The graph $\Gamma$ consists of $n / d$ disjoint "stars": every sequence of period $n$ is connected to the sequence of period $n / d$ obtained by changing the respective $x^{\prime}$ to $x$. Every sequence of period $n$ is hence a vertex of $\Gamma$ of valence 1 and every sequence of period $n / d$ is a vertex of valence $d$. Consequently, paths without back-tracking have length at most 2 and the labels read on these paths are the elements described in the lemma. The rest of the lemma easily follows.

Proposition 3.3. Let $e_{1}, \ldots e_{n}$ be a free basis of the group $\mathbb{Z}^{n}$. The map $\rho: F_{n} \rightarrow \mathbb{Z}^{n}$ given by $a_{i} \mapsto e_{i}$ can be extended to a homomorphism $\rho: \Omega(v) \rightarrow \mathbb{Z}^{n}$. Consequently, $\Omega(v) / \Omega(v)^{\prime} \cong \mathbb{Z}^{n}$.

Proof. Write $\pi: F_{n} \rightarrow \Omega(v)$ the natural quotient map. Assume by contradiction that $\rho$ does not factor through $\pi$; then there exists $g \in \operatorname{ker} \pi \backslash \operatorname{ker} \rho$. Let $g=$ $a_{i_{1}}^{ \pm 1} a_{i_{2}}^{ \pm 1} \ldots a_{i_{k}}^{ \pm 1}$ be a shortest (i.e., one with smallest $k$ ) element of $F_{n}$ in ker $\pi \backslash \operatorname{ker} \rho$. Clearly we may assume $g$ is not contained in the nucleus, since every non-trivial word of length $\leq 2$ has an active state, and is therefore non-trivial in $\operatorname{aut}\left(X^{*}\right) / \operatorname{aut}\left(X^{*}\right)^{\prime}$.

Let us write $\Psi(g)=\left\langle\left\langle g_{0}, g_{1}\right\rangle\right\rangle$. Then, by considering the formulae, we see that the sum of the lengths of $g_{0}$ and $g_{1}$ is at most equal to $k$; it could be shorter, if some cancellation inside $g_{0}$ or $g_{1}$ occurs. The formulae (1) also show that $\rho\left(g_{0}\right)+\rho\left(g_{1}\right)=$ $T(\rho(g))$, where $T: \mathbb{Z}^{n} \rightarrow \mathbb{Z}^{n}$ is the isomorphism $e_{i} \mapsto e_{i-1 \bmod n}$. Consequently, either $\rho\left(g_{0}\right)$ or $\rho\left(g_{1}\right)$ is non-trivial. Since $\Omega(v)$ is contracting, $g_{0}$ and $g_{1}$ are shorter than $g$, and we have contradicted the minimality of $g$.

Lemma 3.4. Define the subgroups $\varepsilon_{i} \leq F_{n}$ inductively by $\varepsilon_{0}=1$ and

$$
\varepsilon_{i+1}=\Psi^{-1}\left(\varepsilon_{i} \times \varepsilon_{i}\right)
$$


Then $\varepsilon_{\infty}=\bigcup_{i=1}^{\infty} \varepsilon_{i}$ is the kernel of the natural homomorphism $\pi: F_{n} \rightarrow \Omega(v)$.

Proof. By contraction, for every $g \in F_{n}$ there exists $k$ such that all coordinates of $\Psi^{k}(g)$ belong to the nucleus. The intersection of the nucleus with $\operatorname{ker}(\pi)$ is trivial. Therefore, if $g$ belongs to $\operatorname{ker}(\pi)$, then there exists $k$ such that all the coordinates of $\Psi^{k}(g)$ are trivial, i.e., $g \in \operatorname{ker} \Psi^{k}=\varepsilon_{k}$.

3.2. Endomorphisms. Let us define the following endomorphism $\varphi$ of the free group $F_{n}$ :

$$
\varphi\left(a_{n}\right)=a_{1}^{2}, \quad \varphi\left(a_{i}\right)= \begin{cases}a_{i+1} & \text { if } x_{i}=0, \\ a_{1} a_{i+1} a_{1}^{-1} & \text { if } x_{i}=1,\end{cases}
$$

when $1 \leq i<n$.

We will also write $\varphi$ for its image in $\Omega(v)$. It follows directly from the definition that

$$
\varphi\left(a_{i}\right)=\left\langle\left\langle a_{i}, 1\right\rangle\right\rangle \text { when } 1 \leq i<n, \quad \varphi\left(a_{n}\right)=\left\langle\left\langle a_{n}, a_{n}\right\rangle\right\rangle .
$$

Proposition 3.5. The substitution $\varphi$ induces an endomorphism of the group $\mathbb{R}(v)$ such that

$$
\varphi(g)=\left\langle\left\langle g, \rho_{n}(g)\right\rangle\right\rangle,
$$

where $\rho_{n}$ is the composition of the abelianization $a_{i} \mapsto e_{i} \in \mathbb{Z}^{n}$ and the projection $e_{n} \mapsto a_{n}, e_{i} \mapsto 1$ for $i \neq n$.

Lemma 3.6. For every $\ell \geq 1$ we have

$$
\mathcal{E}_{\ell+1} \leq \mathcal{E}_{1} \cdot \varphi\left(\mathcal{E}_{\ell}\right) \cdot a_{1}^{-1} \varphi\left(\mathcal{E}_{\ell}\right) a_{1} .
$$

Proof. Consider $g \in \mathcal{E}_{\ell+1}$. Then $\Psi(g)=\left\langle\left\langle g_{0}, g_{1}\right\rangle\right\rangle$, with $g_{0}, g_{1} \in \mathcal{E}_{\ell}$. We have $\Psi\left(\varphi\left(g_{i}\right)\right)=\left\langle\left\langle g_{i}, \rho_{n}\left(g_{i}\right)\right\rangle\right\rangle$ for all $i \in\{0,1\}$. Moreover, $\rho_{n}\left(g_{i}\right)=1$, since $g_{i}=1$ in $\Re(v)$ and the image of $\rho$ is free. Hence,

$$
\Psi(g)=\left\langle\left\langle g_{0}, g_{1}\right\rangle\right\rangle=\Psi\left(\varphi\left(g_{0}\right) \cdot a_{1}^{-1} \varphi\left(g_{1}\right) a_{1}\right),
$$

so $g=h \cdot \varphi\left(g_{0}\right) \cdot a_{1}^{-1} \varphi\left(g_{1}\right) a_{1}$ for some $h \in \mathcal{E}_{1}$.

3.3. L-presentation. We give a recursive presentation of $\Omega(v)$ by generators and relations.

For $r \geq 0$ define the set of commutators, following [1]

$$
\begin{aligned}
\mathcal{R}_{r}=\left\{\left[a_{i}, a_{j}^{a_{1}^{2 k}}\right] \mid 2 \leq i, j \leq n, 0 \leq 2 k \leq r, x_{i-1} \neq x_{j-1}\right\} \\
\cup\left\{\left[a_{i}, a_{j}^{a_{1}^{2 k+1}}\right] \mid 2 \leq i, j \leq n, 0 \leq 2 k+1 \leq r, x_{i-1}=x_{j-1}\right\} .
\end{aligned}
$$


Lemma 3.7. The subgroup $\mathcal{E}_{1}<F_{n}$ is the normal closure of $\mathcal{R}_{\infty}=\bigcup_{r \geq 0} \mathcal{R}_{r}$.

Proof. It is easy to see that $\mathcal{R}_{r} \subset \mathcal{E}_{1}$ for all $r$. Let now $g \in \mathcal{E}_{1}$ be any non-trivial reduced group word. We can write it in the form $a_{1}^{k} c_{1} c_{2} \ldots c_{m}$, where $c_{s}=a_{i_{s}}^{ \pm a_{1}^{j_{s}}}$ for some $i_{s} \in\{2, \ldots, n\}$ and some $j_{s} \in \mathbb{Z}$. Since $g=1$ in $\Re(v)$, we have $k=0$ by Proposition 3.3. Let us write $\Psi(g)=\left\langle\left\langle g_{0}, g_{1}\right\rangle\right\rangle$. Then each of $g_{0}, g_{1}$ respectively is the product of some $a_{i_{s}-1}^{ \pm a_{n}^{k_{s}}}$, where $\left|2 k_{s}-j_{s}\right| \leq 1$ and only those $s$ with $j_{s}$ congruent modulo 2, respectively not congruent, to $x_{i_{s}-1}$, are selected.

Now since $g_{0}=g_{1}=1$, there must exist $s<t$ such that $i_{s}=i_{t}$ and $k_{s}=k_{t}$ and $c_{s}, c_{t}$ have opposing signs, both of them occurring in the same $g_{i}$, and none of the $c_{s+1}, \ldots, c_{t-1}$ contributing to that $g_{i}$. Then the relations in $\mathcal{R}_{\infty}$ allow the commutation of $c_{s}$ with $c_{s+1}, \ldots, c_{t-1}$, and its eventual cancellation with $c_{t}$. One then proceeds by induction on the length of $g$.

Theorem 3.8. The group $\Re(v)$ has the following presentation:

$$
\left.\Re(v)=\left\langle a_{1}, \ldots a_{n}\right| \varphi^{\ell}\left(\mathcal{R}_{2}\right) \text { for all } \ell \geq 0\right\rangle,
$$

where $\varphi$ and $\mathcal{R}_{2}$ and given respectively in (2) and (3).

Proof. In view of Lemmata 3.4,3.6 and 3.7, it suffices to show for all $r \geq 0$ that $\mathcal{R}_{r}$ is a consequence of $\mathcal{R}^{*}=\bigcup_{k \geq 0} \varphi^{k}\left(\mathcal{R}_{2}\right)$. Consider therefore a relation $w_{i j k}=\left[a_{i}, a_{j}^{a_{1}^{k}}\right]$ with $k \geq 3$. We write $g \equiv h$ to mean that they are equivalent under relations in $\mathcal{R}^{*}$.

We first write $a_{j}^{a_{1}^{k}}=a_{j}^{a_{1}^{k-2}}\left[a_{j}, a_{1}^{2}\right]^{a_{1}^{k-2}}$; then by $[a, b c]=[a, c][a, b]^{c}$ the relation $w_{i j k}$ follows from the relations $w_{i j(k-2)}$ and $\left[a_{i},\left[a_{j}, a_{1}^{2}\right]_{1}^{a_{1}^{k-2}}\right]$.

We then note that $\left[a_{p},\left[a_{p}, a_{1}\right]\right] \in \mathcal{R}_{2}$ for all $p$, and more generally $\left[a_{p},\left[a_{p}, a_{q}\right]\right] \in$ $\mathcal{R}^{*}$ for all $p \geq q$ : if $x_{p-1}=x_{q-1}$, this relation is obtained as $\varphi\left(\left[a_{p-1},\left[a_{p-1}, a_{q-1}\right]\right]\right)$, while if $x_{p-1} \neq x_{q-1}$, it follows from $\left[a_{p}, a_{q}\right] \in \mathcal{R}_{2}$. In particular $\left[a_{n},\left[a_{n}, a_{i-1}\right]\right] \in$ $\mathcal{R}^{*}$, and $\left[a_{1}^{2},\left[a_{1}^{2}, a_{i}\right]\right] \in \mathcal{R}^{*}$. This allows to reduce $\left[a_{i},\left[a_{j}, a_{1}^{2}\right]_{1}^{a_{1}^{k-2}}\right]$ to $\left[a_{i},\left[a_{j}, a_{1}^{2}\right]^{e}\right]$ for some $e \in\left\{1, a_{1}^{-1}\right\}$. Now if $e=1$ then we have $\left[a_{i}, a_{j}, a_{1}^{2}\right] \equiv w_{i j 2}\left[a_{i}, a_{j}\right]$, and if $e=a_{1}^{-1}$ then we have $\left[a_{i},\left[a_{j}, a_{1}^{2}\right]^{a_{1}^{-1}}\right]=w_{i j 1}^{-a_{1}^{-1}} w_{i j 1}^{-a_{1}^{-1+2 a_{j}-1}}$.

Since the endomorphism $\varphi$ is injective, we can embed the group $\Omega(v)$ into its ascending $\mathrm{HNN}$-extension by $\varphi$, i.e., in the group generated by $\Omega(v)$ and an element $t$ whose action by conjugation on $\Re(v)$ coincides with $\varphi$.

Setting $a=a_{1}^{-1}$, we have a new generating system $\left\{a, a^{t}, a^{t^{2}}, \ldots, a^{t^{n-1}}\right\}$ of the group $\Omega(v)$; the identification is

$$
\begin{aligned}
& a_{i+1}=a^{x_{i}} a_{i}^{t} a^{-x_{i}}=a^{x_{i}} a^{x_{i-1} t} \ldots a^{x_{1} t^{i-1}} a^{-t^{i}} a^{-x_{1} t^{i-1}} \ldots a^{-x_{i-1} t} a^{-x_{i}}, \\
& a^{t^{i}}=a_{i+1}^{-a^{x_{i}+x_{i-1}} t+\cdots+x_{1} t^{i-1}} .
\end{aligned}
$$


The last theorem then yields

Theorem 3.9. Write $p(t)=x_{n-1} t+x_{n-2} t^{2}+\cdots+x_{1} t^{n-1} \in \mathbb{Z}[t]$. Then the group $\Omega(v)$ is isomorphic to the subgroup $\left\langle a, a^{t}, a^{t^{2}}, \ldots, a^{t^{n-1}}\right\rangle$ of the finitely presented group

$$
\left.\langle a, t| a^{t^{n}-2 a^{p(t)}},\left[a^{t^{i}}, a^{t^{j} a}\right],\left[a^{t^{i}}, a^{t^{j}} a^{3}\right] \text { for all } 1 \leq i, j<n\right\rangle .
$$

Proof. The first relation is $a_{n}^{t}=a_{1}^{2}$. The others are obtained by rewriting $\left[a_{i}, a_{j}^{a_{1}^{k}}\right]$ for $0 \leq k \leq 2$ in terms of $a$ and $t$. Indeed if $x_{i}=x_{j}$ then the relation $\left[a_{i+1}, a_{j+1}^{a_{1}}\right]$ yields

$$
\begin{aligned}
& {\left[a^{x_{i-1} t} \ldots a^{x_{1} t^{i-1}} a^{-t^{i}} a^{-x_{1} t^{i-1}} \ldots a^{-x_{i-1} t}\right.} \\
& \left.\quad\left(a^{x_{j-1} t} \ldots a^{x_{1} t^{j-1}} a^{-t^{j}} a^{-x_{1} t^{j-1}} \ldots a^{-x_{j-1} t}\right)^{a_{1}}\right]
\end{aligned}
$$

which is equivalent to $\left[a^{t^{i}}, a^{t^{j}} a\right]$ for all $i, j$. If $x_{i} \neq x_{j}$, then the relation $\left[a_{i+1}, a_{j+1}\right]$ gives the same commutation relation as above, while $\left[a_{i+1}, a_{j+1}^{a_{1}^{2}}\right]$ gives the equivalent form $\left[a^{t^{i}}, a^{t^{j}} a^{3}\right]$.

3.4. Torsion. We show that $\Re(v)$ is torsion-free for all $v$ :

Proposition 3.10. Let $\rho: \Omega(v) \rightarrow \Omega(v) / \Omega(v)^{\prime} \cong \mathbb{Z}^{n}$ be the canonical epimorphism. An element $g \in \Re(v)$ is level-transitive if and only if all coordinates of $\rho(g)$ are odd.

Proof. We write $\rho\left(a_{i}\right)=e_{i}$, where $\left(e_{i}\right)$ is a free basis of $\mathbb{Z}^{n}$. Recall that aut $\left(X^{*}\right) /$ aut $\left(X^{*}\right)^{\prime}$ is $\left(\frac{\mathbb{Z}}{2 \mathbb{Z}}\right)^{\omega}$, the identification being $\tau: g \mapsto\left(i_{0}, i_{1}, \ldots\right) \in\left(\frac{\mathbb{Z}}{2 \mathbb{Z}}\right)^{\omega}$ where $i_{m}$ is the parity of the number of active restrictions $\left.g\right|_{v}$ for all $v \in X^{m}$. A tree automorphism is active if it acts non-trivially on the first level.

It is well known that $g \in \operatorname{aut}\left(X^{*}\right)$ is level-transitive if and only if $\tau(g)=$ $(1,1, \ldots)$. Now if $g \in \Omega(v)$, then $\tau(g)_{m}$ is the parity of the exponent of $e_{m+1 \bmod n}$ in $\rho(g)$.

Proposition 3.11. The group $\Re(v)$ is torsion free.

Proof. Apply Proposition 2.2 with $N=G^{\prime}$.

3.5. Weak branchness. We show that the groups $\Re(v)$ are weakly branch and residually poly- $\mathbb{Z}$ :

Theorem 3.12. Let $v \in X^{*}$ be a non-empty sequence. Then the group $\Re(v)$ is weakly branch on $\Re(v)^{\prime}$. 
Note that the group $\Re(\emptyset)$ is isomorphic to $\mathbb{Z}$ and so is not weakly branch.

Proof. As a first step, let us check that $\Omega(v)^{\prime} \times \Omega(v)^{\prime} \leq \Omega(v)$. It is easy to check that

$$
\left\langle a_{1}, \ldots, a_{n-1}, a_{1}^{a_{n}}, \ldots, a_{n-1}^{a_{n}}\right\rangle \times\left\langle a_{1}, \ldots, a_{n-1}, a_{1}^{a_{n}}, \ldots, a_{n-1}^{a_{n}}\right\rangle \leq \Omega(v) .
$$

But

$$
\Omega(v)^{\prime} \leq\left\langle a_{1}, \ldots, a_{n-1}, a_{1}^{a_{n}}, \ldots, a_{n-1}^{a_{n}}\right\rangle,
$$

since $\Omega(v)^{\prime}$ is contained in the kernel of the homomorphism $\rho_{n}$. Hence $\Omega(v)^{\prime} \times$ $\Re(v)^{\prime} \leq \Re(v)$.

Let now $g=\left\langle\left\langle g_{0}, g_{1}\right\rangle\right\rangle$ be an arbitrary element of the subgroup $\Omega(v)^{\prime} \times \Omega(v)^{\prime}$. Then $\varphi\left(g_{0}\right)=\left\langle\left\langle g_{0}, \rho_{n}\left(g_{0}\right)\right\rangle\right\rangle \in \Omega(v)^{\prime}$ and $a_{1}^{-1} \varphi\left(g_{1}\right) a_{1}=\left\langle\left\langle\rho_{n}\left(g_{1}\right), g_{1}\right\rangle\right\rangle \in \Omega(v)^{\prime}$, where $\varphi$ and $\rho_{n}$ are as in Proposition 3.5. But $\rho_{n}\left(g_{0}\right)=\rho_{n}\left(g_{1}\right)=1$, since $g_{0}, g_{1} \in$ $\Omega(v)^{\prime}$. Therefore, $g=\varphi\left(g_{0}\right) \cdot a_{1}^{-1} \varphi\left(g_{1}\right) a_{1} \in \Omega(v)^{\prime}$.

It suffices now to check that $\Omega(v)^{\prime}$ is not trivial. For that purpose, note that $\left[a_{1}, a_{2}\right]=\left\langle\left\langle a_{1}^{ \pm a_{n}}, a_{1}^{\mp 1}\right\rangle\right\rangle$ has non-trivial image in $\mathbb{Z}^{n}$.

Proposition 3.13. We have $\Omega(v)^{\prime} /\left(\Omega(v)^{\prime} \times \Omega(v)^{\prime}\right) \cong \mathbb{Z}^{n-1}$.

Proof. Since the elements $\left[a_{i}, a_{j}\right]$ belong to $\Omega(v)^{\prime} \times \Omega(v)^{\prime}$ if $i, j \geq 2$, we see that $\Re(v)^{\prime} / \Omega(v)^{\prime} \times \Re(v)^{\prime}$ is abelian and generated by the $\left[a_{1}, a_{i+1}\right]$ for $i \in\{1, \ldots, n-1\}$.

We then have $\left[a_{1}, a_{i+1}\right]=\left\langle\left\langle a_{i}^{\mp a_{n}}, a_{i}^{ \pm 1}\right\rangle\right\rangle$; by Proposition 3.3, these are independent in $\left(\Omega(v) / \Omega(v)^{\prime}\right)^{2}$.

It follows that the groups $\Re(v)$ are not branch; otherwise, they would be branch on a subgroup $K$ with $\Omega(v)^{\prime} \leq K \leq \Omega(v)$, the last inclusion of finite index. Then $K / \Omega(v)^{\prime}$ has rank $n$, so $K /\left(\Re(v)^{\prime} \times \Re(v)^{\prime}\right)$ has Hirsch length $2 n-1$, but it contains $(K \times K) /\left(\Omega(v)^{\prime} \times \Omega(v)^{\prime}\right)$ of Hirsch length $2 n$, a contradiction.

It follows that $\Omega(v)$ admits arbitrarily large poly- $\mathbb{Z}$ quotients: the successive quotients along the descending series $\Omega(v)>\Omega(v)^{\prime}>\Omega(v)^{\prime} \times \Omega(v)^{\prime}>\cdots$ are all free abelian.

Corollary 3.14. The group $\Re(v)$ is left orderable.

\section{The groups $\Re(w, v)$}

Let $w=y_{1} \ldots y_{k} \in X^{*}$ and $v=x_{1} \ldots x_{n} \in X^{*}$ be a pair of non-empty words such that $y_{k} \neq x_{n}$. We denote by $\Omega(w, v)$ the subgroup of aut $\left(X^{*}\right)$ generated by the elements $b_{1}, \ldots b_{k}, a_{1}, \ldots, a_{n}$ defined by

$$
b_{1}=\sigma, \quad b_{j+1}= \begin{cases}\left\langle\left\langle b_{j}, 1\right\rangle\right\rangle & \text { if } y_{j}=0, \\ \left\langle\left\langle 1, b_{j}\right\rangle\right\rangle & \text { if } y_{j}=1,\end{cases}
$$


when $1 \leq j<k$, and

$$
a_{1}=\left\{\begin{array}{ll}
\left\langle\left\langle b_{k}, a_{n}\right\rangle\right\rangle & \text { if } y_{k}=0 \text { and } x_{n}=1, \\
\left\langle\left\langle a_{n}, b_{k}\right\rangle\right\rangle & \text { if } y_{k}=1 \text { and } x_{n}=0,
\end{array} \quad a_{i+1}= \begin{cases}\left\langle\left\langle a_{i}, 1\right\rangle\right\rangle & \text { if } x_{i}=0, \\
\left\langle\left\langle 1, a_{i}\right\rangle\right\rangle & \text { if } x_{i}=1,\end{cases}\right.
$$

when $1 \leq i<n$.

In other words, the group $\Omega(w, v)$ is generated by the automaton whose Moore diagram is shown on Figure 2. In this diagram, only the edges leading to non-trivial states are drawn, and the active state $b_{1}$ is labelled by $\sigma$.

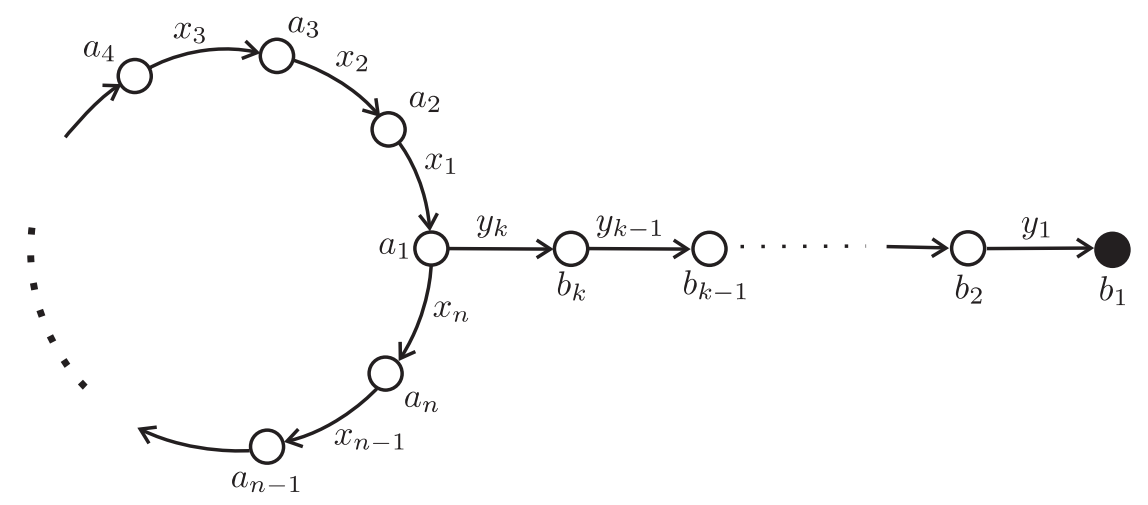

Figure 2. Automaton generating $\Omega(w, v)$.

Let us denote by $v^{\prime}$ the word obtained from the word $v$ by changing 0 to 1 and 1 to 0 . Recall from Section 3 that the automorphism $\alpha \in \operatorname{aut}\left(X^{*}\right)$ defined by the recursion $\alpha=\langle\langle\alpha, \alpha\rangle\rangle \sigma$ exchanges $v$ and $v^{\prime}$, and conjugates $\Omega(v)$ into $\Re\left(v^{\prime}\right)$. Similarly, the groups $\Re(w, v)$ and $\Omega\left(w^{\prime}, v^{\prime}\right)$ are conjugate under $\alpha$.

By convention, let us accept that $b_{k+1}=a_{n+1}=a_{1}$.

Lemma 4.1. The group $\Re(w, v)$ is recurrent and level-transitive.

Proof. The projections of the $a_{i+1}, a_{i+1}^{b_{1}}, b_{j+1}$ and $b_{j+1}^{b_{1}}$, all fixing the first level of $X^{*}$, give all generators; the action is therefore recurrent.

To check that the action of a recurrent group is level-transitive, it suffices to check that it is transitive on the first level; this is achieved by $b_{1}$.

4.1. Wreath recursion. We aim to give a recursive presentation of $\Omega(w, v)$ by generators and relations. Until the end of this section, we assume that $(k, n) \neq(1,1)$; indeed if $k=n=1$ then $\Omega(w, v)$ is infinite dihedral, and has to be taken care of separately. 
Proposition 4.2. The generators $a_{i}, b_{j}$ of $\Omega(w, v)$ have order 2 , and the abelianization $\Omega(w, v) / \Omega(w, v)^{\prime}$ is $\left(\frac{\mathbb{Z}}{2 \mathbb{Z}}\right)^{k+n}$, generated by the images of the $a_{i}$ and $b_{j}$.

Proof. It is clear that the generators are involutions. The image of $\Omega(w, v)$ in $\operatorname{aut}\left(X^{*}\right) / \operatorname{aut}\left(X^{*}\right)^{\prime}$ is generated by the infinite pre-periodic vectors $(0, \ldots, 1, \overline{0})$ corresponding to the images of $b_{j}$, and by $(0, \ldots, 0, \overline{0, \ldots, 1, \ldots, 0})$ corresponding to the images of $a_{i}$; these $k+n$ vectors are linearly independent.

Let $d$ be maximal such that $v=u^{d}$ for some $u \in X^{*}$. We have then, considering indices modulo $n$,

$$
\left.\Psi\left(\left[a_{i}, a_{i+j(n / d)}\right]\right)=\left\langle\left\langle 1,\left[a_{i-1}, a_{i-1+j(n / d)}\right]\right\rangle\right\rangle \quad \text { or } \quad\left\langle\left[a_{i-1}, a_{i-1+j(n / d)}\right], 1\right\rangle\right\rangle,
$$

depending on whether $x_{i-1}=x_{i-1+j(n / d)}$ or not. It follows that $a_{i}$ and $a_{i+j(n / d)}$ commute for every $i \in\{1, \ldots, n\}$ and every $j \in\{1, \ldots, d-1\}$.

Let $F$ be the group given by the presentation

$$
\begin{aligned}
& F=\left\langle a_{1}, \ldots, a_{n}, b_{1}, \ldots, b_{k}\right| \\
& \left.\qquad a_{i}^{2}, b_{j}^{2},\left[a_{i}, a_{i+\ell(n / d)}\right] \text { for all } 1 \leq i \leq n, 1 \leq j \leq k, 1 \leq \ell<d\right\rangle .
\end{aligned}
$$

It is the free product of $k$ copies of $\frac{\mathbb{Z}}{2 \mathbb{Z}}$ and $n / d$ copies of $\left(\frac{\mathbb{Z}}{2 \mathbb{Z}}\right)^{d}$. Let us denote the subgroup $\left\langle a_{i}, a_{i+n / d}, \ldots, a_{i+(d-1) n / d}\right\rangle \cong\left(\frac{\mathbb{Z}}{2 \mathbb{Z}}\right)^{d}$ by $A_{i}$.

Let $\Psi: F \rightarrow F>\Im_{2}$ be given by the recursive definition of the group $\Re(w, v)$, i.e.,

$$
\Psi\left(b_{1}\right)=\sigma, \quad \Psi\left(b_{j+1}\right)= \begin{cases}\left.\left\langle b_{j}, 1\right\rangle\right\rangle & \text { if } y_{j}=0, \\ \left\langle 1, b_{j}\right\rangle & \text { if } y_{j}=1,\end{cases}
$$

when $1 \leq j<k$, and

$$
\Psi\left(a_{1}\right)=\left\{\begin{array}{ll}
\left.\left\langle b_{k}, a_{n}\right\rangle\right\rangle & \text { if } x_{n}=1, \\
\left.\left\langle a_{n}, b_{k}\right\rangle\right\rangle & \text { if } x_{n}=0,
\end{array} \quad \Psi\left(a_{i+1}\right)= \begin{cases}\left\langle\left\langle a_{i}, 1\right\rangle\right\rangle & \text { if } x_{i}=0, \\
\left\langle\left\langle 1, a_{i}\right\rangle\right\rangle & \text { if } x_{i}=1,\end{cases}\right.
$$

when $1 \leq i<n$.

We can iterate the map $\Psi$ to obtain a homomorphism $\left.\Psi^{k}: F \rightarrow F \prec \Im_{2} \prec \cdots\right\urcorner \Im_{2}$, where $\widetilde{S}_{2}$ appears $k$ times in the iterated permutational wreath product.

Lemma 4.3. The wreath recursion $\Psi$ is contracting. Its nucleus is $\left\{b_{1}, \ldots, b_{k}\right\} \cup$ $\bigcup A_{i}$.

Proof. Let us denote by $\|g\|$ the minimal number of generators $a_{i}$ in a representation of $g$ as a product of generators.

It follows from the definition of the recursion $\Psi$ that for every $g=\left\langle\left\langle g_{0}, g_{1}\right\rangle\right\rangle \sigma^{s} \in$ $\Re(w, v)$ we have $\|g\| \geq\left\|g_{0}\right\|+\left\|g_{1}\right\|$. As in the proof of Lemma 3.2, we say that 
$g \in \Re(w, v)$ is stable if there exists an infinite sequence $w_{g}$ (the kneading sequence of $g$ ) such that for every $l$ we have $\|g\|=\left\|\left.g\right|_{v_{l}}\right\|$ where $v_{l}$ is the beginning of length $l$ of $w_{g}$. It also follows that for any word $v \in X^{l}$ different from $v_{l}$ the restriction $\left.g\right|_{v}$ belongs to $\left\langle b_{i}\right\rangle_{i=1, \ldots, k}$, and hence that there exists $m \in \mathbb{N}$ such that $\left.g\right|_{v w}=1$ for all $w \in X^{m}$.

Consequently, if we prove that the set of stable elements is finite, then we show that the recursion is contracting and the nucleus is the set of stable elements and their restrictions.

As in the proof of Lemma 3.2, consider the set $K$ of kneading sequences of the (stable) generators $a_{i}$, and the corresponding graph $\Gamma$. Here also the labels along the paths of the graph $\Gamma$ correspond to the stable elements.

The kneading sequence of $a_{i}$ is equal to $w_{a_{i}}=\left(x_{i-1} x_{i-2} \ldots x_{1} x_{n} x_{n-1} \ldots x_{i}\right)^{\omega}$. If $d$ is as above, then the kneading sequence of $a_{i}$ is of period $n / d$ and the graph $\Gamma$ consists of $n / d$ vertices with $d$ loops attached to every vertex. The labels of the loops attached to vertex $w_{a_{i}}$ are $a_{i}, a_{i+n / d}, \ldots, a_{i+(d-1) n / d}$. Consequently, every stable element belongs to the finite group $A_{i}$ for some $i \in\{1, \ldots, n / d\}$. The rest of the lemma follows.

Lemma 4.4. Define the subgroups $\varepsilon_{\ell} \leq F$ inductively by $\varepsilon_{0}=1$ and

$$
\varepsilon_{\ell+1}=\Psi^{-1}\left(\varepsilon_{\ell} \times \varepsilon_{\ell}\right) .
$$

Then $\mathcal{E}_{\infty}=\bigcup_{\ell=1}^{\infty} \varepsilon_{\ell}$ is the kernel of the natural homomorphism $F \rightarrow \Omega(w, v)$.

Proof. Similar to Lemma 3.4.

4.2. Endomorphisms. In this subsection we define, for each $\Omega(w, v)$, an endomorphism $\varphi$ of $F$, and elements $s, t \in F$ and $m \in\{1,2\}$, with the following meaning: $\Psi\left(a_{1}^{t}\right)=\left\langle\left\langle b_{k}, a_{n}^{s}\right\rangle\right\rangle$; in $\Omega(w, v)$ the subgroup $\left\langle a_{n}^{s}, b_{k}\right\rangle$ is dihedral of order $2^{m+1}$; and the subgroup $\left\langle a_{1}^{t}, b_{1}\right\rangle$ is dihedral of order $2^{m+2}$; and $\varphi(s) \in\left\{t, b_{1} t\right\}$. We use these to construct an endomorphism of $\Omega(w, v)$, considering different cases in turn and taking care of small values of $k$ and $n$.

Assume first that $k, n \geq 2$. Then $\left[a_{n}^{s}, b_{k}\right]$ is trivial in $\Re(w, v)$ for at least one value of $s \in\left\langle b_{1}\right\rangle$. Let $t \in\left\langle b_{1}, b_{2}\right\rangle$ be such that $\Psi\left(a_{1}^{t}\right)=\left\langle\left\langle b_{k}, a_{n}^{s}\right\rangle\right\rangle$. Set $m=1$.

Assume next that $k \geq 3$ and $n=1$. Then there exists $r \in\left\langle b_{1}\right\rangle$ such that $\left[b_{k}^{r}, b_{k-1}\right]=1$ in $\Omega(w, v)$; so there exists $s \in\left\langle b_{1}, b_{2}\right\rangle$ such that $\left[a_{1}^{s}, b_{k}\right]=1$ in $\Re(w, v)$; so there exists $t \in\left\langle b_{1}, b_{2}, b_{3}\right\rangle$ such that $\Psi\left(a_{1}^{t}\right)=\left\langle\left\langle b_{k}, a_{1}^{s}\right\rangle\right\rangle$. Set also $m=1$.

Assume next that $k=2$ and $n=1$. Then there exists $r, s \in\left\langle b_{1}\right\rangle$ such that $b_{2}^{r}=\left\langle\left\langle b_{1}, 1\right\rangle\right\rangle$ in $\Omega(w, v)$ and $a_{1}^{r s}=\left\langle\left\langle b_{2}, a_{1}\right\rangle\right\rangle$ in $\Omega(w, v)$; so there exists $t \in\left\langle b_{1}, b_{2}\right\rangle$ such that $a_{1}^{t}=\left\langle\left\langle b_{2}, a_{1}^{s}\right\rangle\right\rangle$. Set $m=2$.

Finally, consider the case $k=1$ and $n \geq 2$. Set $s=1$, and let $t \in\left\langle b_{1}\right\rangle$ be such that $a_{1}^{t}=\left\langle\left\langle b_{1}, a_{n}\right\rangle\right\rangle$. Set $m=2$. 
Now define the endomorphism $\varphi$ of $F$ by

$$
\begin{aligned}
& \varphi\left(b_{j}\right)=\left\{\begin{array}{ll}
b_{j+1} & \text { if } y_{j}=0, \\
b_{j+1}^{b_{1}} & \text { if } y_{j}=1,
\end{array} \text { when } 1 \leq j<k, \quad \varphi\left(b_{k}\right)=a_{1}^{t},\right. \\
& \varphi\left(a_{i}\right)=\left\{\begin{array}{ll}
a_{i+1} & \text { if } x_{i}=0, \\
a_{i+1}^{b_{1}} & \text { if } x_{i}=1,
\end{array} \text { when } 1 \leq i<n,\right.
\end{aligned}
$$

We then have

$$
\begin{aligned}
& \Psi \varphi\left(b_{j}\right)=\left\langle\left\langle b_{j}, 1\right\rangle\right\rangle \text { when } 1 \leq j<k, \quad \Psi \varphi\left(b_{k}\right)=\left\langle\left\langle b_{k}, a_{n}^{s}\right\rangle\right\rangle, \\
& \Psi \varphi\left(a_{i}\right)=\left\langle\left\langle a_{i}, 1\right\rangle\right\rangle \text { when } 1 \leq i<n, \quad \Psi \varphi\left(a_{n}\right)=\left\langle\left\langle a_{n}, b_{k}\right\rangle\right\rangle .
\end{aligned}
$$

Proposition 4.5. The endomorphism $\varphi: F \rightarrow F$ induces an endomorphism of the group $\Re(w, v)$ such that

$$
\varphi(g)=\langle\langle g, \rho(g)\rangle\rangle,
$$

where $\rho$ is an endomorphism with finite image; more precisely, $\rho\left(b_{j}\right)=\rho\left(a_{i}\right)=1$ for $j<k$ and $i<n$; and $\rho\left(b_{k}\right)=a_{n}^{s}$ and $\rho\left(a_{n}\right)=b_{k}$, so that $\rho(F)$ is dihedral of order $2^{m+1}$.

Proof. It is clear that $\rho$ is an endomorphism if $m=1$, since then it factors through $\Omega(w, v) \rightarrow \operatorname{aut}\left(X^{*}\right) / \operatorname{aut}\left(X^{*}\right)^{\prime}$.

Consider then the case $k=1$. The map $\rho$ then factors through $\Omega(w, v) \rightarrow$ $\Omega(w, v)>\mathfrak{S}_{2} \rightarrow\left(\operatorname{aut}\left(X^{*}\right) / \operatorname{aut}\left(X^{*}\right)^{\prime}\right)>\mathfrak{S}_{2}$.

Consider finally the case $k=2, n=1$. The map $\rho$ can then be seen, by direct calculation, to factor through $\Re(w, v) \rightarrow \operatorname{aut}\left(X^{4}\right)$.

Define now $\bar{F}=F /\left\langle\varphi^{\ell}\left(a_{n}^{s} b_{k}\right)^{2^{m}} \mid \ell \in \mathbb{N}\right\rangle$, and denote by $\bar{\varepsilon}_{\ell}$ the image of $\varepsilon_{\ell}$ in $\bar{F}$. Again $\varphi$ induces an endomorphism of $\bar{F}$. Note that $\bar{F} \rightarrow \Omega(w, v)$ maps isomorphically $\left\langle a_{n}^{s}, b_{k}\right\rangle$ and $\left\langle a_{1}^{t}, b_{1}\right\rangle$.

Lemma 4.6. For every $\ell \geq 1$ we have

$$
\overline{\varepsilon_{\ell+1}} \leq \overline{\varepsilon_{1}} \cdot \varphi\left(\overline{\varepsilon_{\ell}}\right) \cdot b_{1} \varphi\left({\overline{\varepsilon_{i}}}\right) b_{1}
$$

Proof. The proof is similar to that of Lemma 3.6. Consider $g \in \overline{\varepsilon_{\ell+1}}$. Then $\Psi(g)=$ $\left\langle\left\langle g_{0}, g_{1}\right\rangle\right\rangle$, with $g_{0}, g_{1} \in \overline{\varepsilon_{\ell}}$. We have $\Psi \varphi\left(g_{i}\right)=\left\langle\left\langle g_{i}, \rho\left(g_{i}\right)\right\rangle\right\rangle$ for all $i \in\{0,1\}$, where $\rho$ is the endomorphism with finite image given by Proposition 4.5. Moreover, $\rho\left(g_{i}\right)=1$ in $\bar{F}$, since $\rho\left(g_{i}\right)$ is a relation in $\Re(w, v)$. Hence,

$$
\Psi(g)=\left\langle\left\langle g_{0}, g_{1}\right\rangle\right\rangle=\Psi\left(\varphi\left(g_{0}\right) \cdot b_{1} \varphi\left(g_{1}\right) b_{1}\right),
$$

and $g=h \cdot \varphi\left(g_{0}\right) \cdot b_{1} \varphi\left(g_{1}\right) b_{1}$ for some $h \in \overline{\mathcal{E}_{1}}$. 
4.3. L-presentation. Let $O$ denote the elements of $\left\langle a_{1}^{t}, b_{1}\right\rangle<\bar{F}$ that contain an odd number of $b_{1}$ 's, and let $E$ denote those elements that contain an even number of $b_{1}$ 's. Both of these sets are finite. Define then

$$
\begin{aligned}
& \mathcal{R}=\{ {\left.\left[b_{i}, b_{j}^{w}\right] \mid 2 \leq i, j \leq k, w \in O \text { if } y_{i-1}=y_{j-1}, w \in E \text { if } y_{i-1} \neq y_{j-1}\right\} } \\
& \cup\left\{\left[a_{i}, b_{j}^{w}\right] \mid 2 \leq i \leq n, 2 \leq j \leq k, w \in O \text { if } x_{i-1}=y_{j-1},\right. \\
&\left.w \in E \text { if } x_{i-1} \neq y_{j-1}\right\} \\
& \cup\left\{\left[a_{i}, a_{j}^{w}\right] \mid 2 \leq i, j \leq n, w \in O \text { if } x_{i-1}=x_{j-1}, w \in E \text { if } x_{i-1} \neq x_{j-1}\right\} .
\end{aligned}
$$

Lemma 4.7. We have $\overline{\varepsilon_{1}} \leq \mathcal{R}^{\bar{F}} \leq \overline{\varepsilon_{\infty}} \leq \bar{F}$.

Proof. It is easy to see that $\mathcal{R} \subset \overline{\mathcal{E}_{\infty}}$. Let now $g \in \overline{\mathcal{E}_{1}}$ be any non-trivial reduced group word. We can write it in the form $h c_{1} c_{2} \ldots c_{m}$, where $c_{s}=a_{i_{s}}^{h_{s}}$ or $=b_{j_{s}}^{h_{s}}$ for some $i_{s}, j_{2} \geq 2$ and some $h, h_{s} \in\left\langle a_{1}^{t}, b_{1}\right\rangle$. Since $g=1$ in $\Omega(v)$, we have $h=1$; this is clear if $a_{1}^{t}$ and $b_{1}$ commute, because then $\left\langle a_{1}^{t}, b_{1}\right\rangle$ embeds in $\Omega(w, v) / \mathscr{R}(w, v)^{\prime}$, by Proposition 4.2. On the other hand, if $h=\left(a_{1}^{t} b_{1}\right)^{2} \neq 1$, then the first projection of $g$ contains precisely two $a_{n}$ 's that do not cancel, and there will exist two vertices such that each of the $a_{n}$ 's projects on one; then these states will be non-trivial, again using Proposition 4.2, so $g \neq 1$, a contradiction.

Let us write $\Psi(g)=\left\langle\left\langle g_{0}, g_{1}\right\rangle\right\rangle$. Then each of $g_{0}, g_{1}$ respectively is the product of some $a_{i_{s}-1}^{k_{s}}$ and $b_{j_{s}-1}^{k_{s}}$, where $k_{s} \in\left\langle a_{n}, b_{k}\right\rangle$ and only those $s$ with $h_{s} \in O$ respectively in $E$, are selected.

Now since $g_{0}=g_{1}=1$, there must exist $s<t$ such that $c_{s}, c_{t}$ are both $b_{*}^{*}$ or both $a_{*}^{*}$ and $h_{s}=h_{t}$, both of them occurring in the same $g_{i}$, and none of the $c_{s+1}, \ldots, c_{t-1}$ contributing to that $g_{i}$. The relations in $\mathcal{R}$ and $\bar{F}$ allow the commutation of $c_{s}$ with $c_{s+1}, \ldots, c_{t-1}$, and its eventual cancellation with $c_{t}$. One then proceeds by induction on the length of $g$.

Theorem 4.8. The group $\Re(w, v)$ has the following presentation:

$$
\left.\Re(w, v)=\left\langle a_{1}, \ldots a_{n}, b_{1}, \ldots, b_{k}\right|\{\text { relations of } \bar{F}\} \cup \varphi^{\ell}(\mathcal{R}) \text { for all } \ell \geq 0\right\rangle,
$$

where $\varphi$ and $\mathcal{R}$ and given respectively before Proposition (4.5) and in (3).

Proof. This follows from Lemmata 4.4, 4.6 and 4.7.

Since the endomorphism $\varphi$ is injective, we can embed the group $\mathbb{R}(w, v)$ into its ascending $\mathrm{HNN}$-extension by $\varphi$, i.e., in the group generated by $\Re(w, v)$ and an element $t$ whose action by conjugation on $\Omega(w, v)$ coincides with $\varphi$.

There is an $u \in\left\langle b_{2}, b_{2}^{b_{1}}, b_{3}, b_{3}^{b_{1}}\right\rangle$ such that $\varphi\left(b_{k}\right)=a_{1}^{u b_{1}^{y_{k}}}$; the group $\left\langle a_{1}^{u}, b_{1}\right\rangle$ is dihedral of order $2^{m+2}$, and we have $\left(a_{1}^{u} b_{1}\right)^{2^{m+1}}=1$. We set $a=a_{1}^{u}$ and $b=b_{1}$ 
to obtain a new generating system $\left\{b, b^{t}, \ldots, b^{t^{k-1}}, a, a^{t}, \ldots, a^{t^{n-1}}\right\}$ of the group $\mathfrak{\Omega}(w, v)$; the identification with $\left\{a_{i}, b_{j}\right\}$ is similar to that in (4). The last theorem then yields

Theorem 4.9. Write $p(t)=x_{n}+x_{n-1} t+\cdots+x_{1} t^{n-1} \in \mathbb{Z}[t]$ and $q(t)=y_{k}+y_{k-1} t+\cdots+y_{1} t^{k-1} \in \mathbb{Z}[t]$. Let $m \in\{1,2\}$ and $u \in\left\langle b^{t}, b^{t b}, b^{t^{2}}, b^{t^{2} b}\right\rangle$ be as above. Then the group $\Omega(w, v)$ is isomorphic to the subgroup $\left\langle a, a^{t}, \ldots, a^{t^{n-1}}, b, b^{t}, \ldots, b^{t^{k-1}}\right\rangle$ of the finitely presented group (where the generator a is redundant)

$$
\begin{aligned}
& \langle a, b, t| a^{2}, b^{2},(a b)^{2^{m+1}}, b^{t^{k}} a^{b^{q(t)}}, a^{u^{-1}\left(t^{n}-b^{p(t)}\right)}, \\
& {\left[a, a^{t^{j n / d}}\right] \text { for all } j \in\{1, \ldots, d-1\},} \\
& {\left[b^{t^{i}}, b^{t^{j}} b(a b)^{2 \ell}\right] \text { for all } 1 \leq i, j<k \text { and } 0 \leq \ell \leq 2^{m} \text {, }} \\
& {\left[a^{t^{i}}, b^{t^{j} b(a b)^{2 \ell}}\right] \text { for all } 1 \leq i<n, 1 \leq j<k \text { and } 0 \leq \ell \leq 2^{m} \text {, }} \\
& \left.\left[a^{t^{i}}, a^{t^{j} b(a b)^{2 \ell}}\right] \text { for all } 1 \leq i, j<n \text { and } 0 \leq \ell \leq 2^{m}\right\rangle \text {. }
\end{aligned}
$$

Proof. The first three relations express $\left\langle a_{1}^{u}, b_{1}\right\rangle$ as a dihedral group. The fourth one is $b_{k}^{t}=a_{1}^{u b_{1}^{y_{k}}}$, and the fifth one is $a_{n}^{t}=a_{1}^{b_{1}^{x_{n}}}$. The next ones are relations in $F$, and the last three rows are the commutation relations of the form $\left[a_{i}, a_{j}^{w}\right]$ and $\left[a_{i}, b_{j}^{w}\right]$ for $w \in E$ or $\in O$.

4.4. Branchness. We see in this subsection that $\Re(w, v)$ is branch as soon as $k>1$ or $n>1$ :

Theorem 4.10. (1) $\Re(w, v)$ is branch on $\Omega(w, v)^{\prime}$ if $k \geq 2$ and $n \geq 2$, or if $k \geq 3$ and $n=1$; we have $\Omega(w, v) / \Omega(w, v)^{\prime} \cong\left(\frac{\mathbb{Z}}{2 \mathbb{Z}}\right)^{k+n}$ generated by $\left\{a_{i}, b_{j}\right\}$ and $\Omega(w, v)^{\prime} /\left(\Omega(w, v)^{\prime} \times \Omega(w, v)^{\prime}\right)=\left(\frac{\mathbb{Z}}{2 \mathbb{Z}}\right)^{k+n-1}$ generated by $\left\{\left[b_{1}, b_{j}\right]_{2 \leq j \leq k}\right.$, $\left.\left[b_{1}, a_{i}\right]_{1 \leq i \leq n}\right\}$.

(2) $\Re(w, v)$ is branch on $L=\left\langle\left[b_{1}, b_{2} a_{1}\right]\right\rangle^{\Re(w, v)}$ if $k=2$ and $n=1$; we have $\Omega(w, v)^{\prime} / L=\left(\frac{\mathbb{Z}}{2 \mathbb{Z}}\right)^{2}$ generated by $\left\{\left[b_{1}, b_{2}\right],\left[b_{2}, a_{1}\right]\right\}$. Set $x=\left[b_{1}, b_{2} a_{1}\right]$; then $L /(L \times L)=\frac{\mathbb{Z}}{4 \mathbb{Z}} \times \frac{\mathbb{Z}}{2 \mathbb{Z}}$, generated by $\left\{x, x^{a_{1}}\right\}$ with the relations $x^{4}=\left(x^{a_{1}}\right)^{4}=$ $x^{2} x^{2 a_{1}}=1$.

(3) $\Re(w, v)$ is branch on $L=\left\langle\left[a_{i}, a_{j}\right]_{1 \leq i<j \leq n},\left[a_{i}, b_{1}\right]_{1 \leq i<n}\right\rangle^{\Re(w, v)}$ if $k=1$ and $n \geq 2$; we have $\Omega(w, v)^{\prime} / L=2$, generated by $\left[a_{n}, b_{1}\right]$, and $L /(L \times L)=$ $\frac{\mathbb{Z}}{4 \mathbb{Z}} \times\left(\frac{\mathbb{Z}}{2 \mathbb{Z}}\right)^{n-2}$, generated by $\left\{\left[a_{i}, b_{1}\right]\right\}$ where $\left[a_{1}, b_{1}\right]$ has order 4 and the other generators have order 2 . smallskip

(4) If $k=n=1$, then $\Re(w, v)$ is infinite dihedral, and is not even weakly branch. 
Proof. We use the endomorphism from Proposition 4.5. In all cases, it is easy to check that $L$ has finite index as claimed, and that $\varphi(L)=L \times 1 \subset \Omega(w, v) \times 1$.

It then remains to check that $\varphi(L) \subset L$. This is obvious if $L=\Omega(w, v)^{\prime}$ is characteristic. If $k=2$ and $n=1$, we obviously have $\varphi\left(\gamma_{3}(\Omega(w, v))\right) \subset$ $\gamma_{3}(\Omega(w, v))$; but we also have $\varphi(x)=\left[b_{2}^{r}, a_{1}^{t} a_{1}^{u}\right] \in \gamma_{3}(\Omega(w, v))$, so $\varphi(L) \subset L$ with $L=\left\langle\gamma_{3}(\Omega(w, v)), x\right\rangle$ as claimed.

If $k=1$ and $n \geq 2$, then $\varphi\left[a_{i}, a_{j}\right]=\left[a_{i+1}^{s}, a_{j+1}^{t}\right]$ for some $s, t \in\left\langle b_{1}\right\rangle$; now $i+1<n$ or $j+1<n$, so $\left[a_{i+1}, a_{j+1}\right] \in L$ and either $\left[a_{i+1}, b_{1}\right] \in L$ and $\left[a_{j+1}, b_{1}\right] \in L$, so $\left[a_{i+1}^{s}, a_{j+1}^{t}\right] \in L$. Similarly $\varphi\left[a_{i}, b_{1}\right]=\left[a_{i+1}^{s}, a_{1}^{t}\right] \in L$.

4.5. Torsion. First, we note that the group $\Re(w, v)$ always contains elements of infinite order:

Proposition 4.11. Every element $x$ which is a product, in any order, of all the generators $a_{i}, b_{j}$ of $\Omega(w, v)$ has infinite order.

Proof. Consider such an $x$. Its image in $\operatorname{aut}\left(X^{*}\right) / \operatorname{aut}\left(X^{*}\right)^{\prime}$ is $(1,1, \ldots)$ so this element acts level-transitively; in particular, it has infinite order.

Proposition 4.12. If $k \neq 1$ or $n \neq 1$, then $\Omega(w, v)$ contains every finite 2-group as a subgroup. In particular, it contains torsion elements of arbitrarily large order.

Proof. This follows directly from Proposition 2.6, since the groups $\Re(w, v)$ are regular branch on a subgroup $L$ containing a torsion element.

If $k \geq 2$ and $n \geq 2$, this torsion element may be chosen as $\left[b_{1}, b_{2}\right]$. If $k=2$ and $n=1$, we may check that $\left(b_{1} a_{1}\right)^{4}$ belongs to $L$ and has order 2 . If $k=1$ and $n \geq 2$ then $\left[b_{1}, a_{1}\right]$ belongs to $L$ and has order 4 .

\section{Kneading sequences and quadratic polynomials}

5.1. Review of results in holomorphic dynamics. Let $f(z)=z^{2}+c$ be a quadratic polynomial. Suppose that the orbit of the critical point 0 under the iterations of $f$ is finite. Such polynomials are called post-critically finite. We distinguish two cases: when 0 belongs to a finite cycle (periodic case) and when it does not, but its orbit is still finite (pre-periodic case).

Recall that if $M \subset \mathbb{C}$ is a connected and closed set with connected complement, then there exists a unique biholomorphic isomorphism $\Phi_{M}$ of the complement $\overline{\mathbb{C}} \backslash M$ with $\{z \in \overline{\mathbb{C}}|| z \mid>R\}$ such that $\Phi_{M}(\infty)=\infty$ and $\Phi_{M}^{\prime}(\infty)=1$. The external ray $\mathbf{R}_{\alpha}$ is the image of the ray $\left\{r \cdot e^{2 \pi i \cdot \alpha} \mid r \in(R, \infty)\right\}$ under $\Phi_{M}^{-1}$. One says that an external ray $\mathbf{R}_{\alpha}$ lands, if the $\operatorname{limit}_{\lim } \operatorname{liR}_{M} \Phi_{M}^{-1}\left(r \cdot e^{2 \pi i \alpha}\right)$ exists. Here and below the 
angle $\alpha$ is considered to be an element of the group $\mathbb{R} / \mathbb{Z}$, i.e., the angles are counted in full turns.

We use known facts about the dynamics of iterations of quadratic maps (see [9], [10], [11]). The filled-in Julia set $K_{c}$ of $z^{2}+c$ is the set of points which do not escape to infinity under iteration, and the Fatou set is the open set $\overline{\mathbb{C}} \backslash \partial K_{c}$. We assume that $z^{2}+c$ is post-critically finite, hence $K_{c}$ is connected. External rays to the Mandelbrot set are called parameter rays, and external rays to $K_{c}$ are called dynamical rays.

It is easy to see that the image of the dynamical ray $\mathbf{R}_{\alpha}$ under the action of $z^{2}+c$ is equal to the ray $\mathbf{R}_{2 \alpha}$.

Suppose first that 0 belongs to a cycle of length $n$ under iteration of $z^{2}+c$. Then $c$ belongs to a hyperbolic component $M_{c}$ of the interior of the Mandelbrot set. For any other point $c_{1}$ of that component, the quadratic polynomial $z^{2}+c_{1}$ also has a unique attracting cycle of length $n$. If $\Phi\left(c_{1}\right)$ denotes the multiplier of this cycle (i.e., the product of derivatives in all points of the cycle), then $\Phi$ is a conformal isomorphism of $M_{c}$ with the open unit disc $\mathbb{D}=\{z \in \mathbb{C}|| z \mid<1\}$. We obviously have $\Phi(c)=0$, hence $c$ is called the center of the hyperbolic component $M_{c}$. The isomorphism $\Phi: M_{c} \rightarrow \mathbb{D}$ extends to a homeomorphism of the boundary of $M_{c}$ with the unit circle. The preimage of 1 under this homeomorphism is called the root of the component $M_{c}$. There exist exactly two angles $\theta$ such that the parameter ray $\mathbf{R}_{\theta}$ lands on the root of $M_{c}$, except for $n=1$, when such ray is unique.

In the dynamical plane, the point $c$ belongs to a Fatou component $U_{c}$, which is periodic with period $n$ under $f$. There is a unique point $r$ on the boundary of $U_{c}$, fixed under the map $f^{n}: U_{c} \rightarrow U_{c}$ (since $\left.f^{n}\right|_{U_{c}}$ is topologically conjugate (via the Böttcher map) to the restriction of $z^{2}$ to $\mathbb{D}$ ). This point and its forward images are called the roots of their corresponding Fatou components.

A parameter ray $\mathbf{R}_{\theta}$ lands on the root of the hyperbolic component $M_{c}$ if and only if the dynamical ray $\mathbf{R}_{\theta}$ lands on the root of the Fatou component $U_{c}$. Moreover, the number $\theta \in \mathbb{R} / \mathbb{Z}$ belongs to a cycle of length $n$ under the doubling map $\alpha \mapsto 2 \alpha$ : $\mathbb{R} / \mathbb{Z} \rightarrow \mathbb{R} / \mathbb{Z}$. In particular the angle $\theta$ is equal to $p /\left(2^{n}-1\right)$ for some integers $p, n$, and the ray $\mathbf{R}_{2^{k} \theta}$ lands at the root of the Fatou component to which $f^{k}(c)$ belongs.

Conversely, for every rational number $\theta \in \mathbb{R} / \mathbb{Z}$ with odd denominator, the parameter ray $\mathbf{R}_{\theta}$ lands on the root of a hyperbolic component $M_{c}$, and if $c$ is the center of the component (i.e., the preimage of 0 under the multiplicator map), then 0 has the same period under $z^{2}+c$ as $\theta$ has under the doubling map, and the dynamical ray $\mathbf{R}_{\theta}$ lands on the root of the Fatou component of $z^{2}+c$ containing $c$.

Suppose now that 0 is pre-periodic. Then $c$ belongs to the boundary of the Mandelbrot set (it is a Misiurewicz point) and there exists a finite set of angles $\theta$ such that the parameter rays $\mathbf{R}_{\theta}$ land on $c$. For each such $\theta$ the external ray $\mathbf{R}_{\theta}$ in the dynamical plane of $z^{2}+c$ lands on $c$. The pre-period of $\theta$ under the doubling map is the same as the pre-period of $c$ under $z^{2}+c$, but the period of $\theta$ may be a multiple of the period of $c$. Here pre-period and period of a point $x$ under a map $f$ are the minimal positive 
integers $k$ and $n$ such that $f^{k+n}(x)=f^{k}(x)$.

For example, the point $c \approx-0.1011+0.9563 i$ is the landing point of the parameter rays $\mathbf{R}_{\alpha}$ for $\alpha=\frac{9}{56}, \frac{11}{56}$ and $\frac{15}{56}$. The point $c$ has pre-period of length 3 and period of length 1 (i.e., it lands on a fixed point). But the angles have period 3, namely

$$
9 / 56 \mapsto 9 / 28 \mapsto 9 / 14 \mapsto 2 / 7 \mapsto 4 / 7 \mapsto 1 / 7 \mapsto 2 / 7 .
$$

The period of $c$ is determined by $\theta$ as the period of the kneading sequence of $\theta$. Let $S_{0}$ be the image in $\mathbb{R} / \mathbb{Z}$ of the interval $[\theta / 2,(1+\theta) / 2]$, and let $S_{1}$ be the image in $\mathbb{R} / \mathbb{Z}$ of $[(1+\theta) / 2,(2+\theta) / 2]$. For every $\alpha \in \mathbb{R} / \mathbb{Z}$, denote by $I_{\theta}(\alpha)$ its $\theta$-itinerary, defined as the sequence $a_{0} a_{1} \ldots$, where

$$
a_{k}= \begin{cases}0 & \text { if } 2^{k} \alpha \in S_{0} \\ 1 & \text { if } 2^{k} \alpha \in S_{1}, \\ * & \text { if } 2^{k} \alpha \in\{\theta / 2,(1+\theta) / 2\}\end{cases}
$$

The itinerary $I_{\theta}(\theta)$ is called the kneading sequence of the point $\theta \in \mathbb{R} / \mathbb{Z}$ and is denoted $\hat{\theta}$.

If $\theta$ is periodic under the doubling map with period of length $n$, then its kneading sequence is of the form $v v \ldots$, where

$$
v=1 x_{2} \ldots x_{n-1} *,
$$

for some $x_{i} \in\{0,1\}$.

If $\theta$ is strictly pre-periodic with a pre-period of length $k$ and period of length $n$, then its kneading sequence is of the form $w v v \ldots$, where $w=1 x_{2} \ldots x_{k}$ and $v$ are some words over $\{0,1\}$ of length $k$ and $n$ respectively with different last letters.

It may happen that $v$ is a proper power. Then the period of the kneading sequence is a factor of the period of $\theta$ under the doubling map. In any case if $\theta$ is pre-periodic (i.e., if its smallest denominator is even), and the parameter ray $\mathbf{R}_{\theta}$ lands on $c$, then the period of $c$ under iteration of $z^{2}+c$ is equal to the period of the kneading sequence $\hat{\theta}$.

5.2. Iterated monodromy groups of quadratic polynomials. Suppose that $w$ is either a kneading sequence of the form $\left(x_{1} \ldots x_{n-1} *\right)^{\omega}$, or a kneading sequence of the form $y_{1} \ldots y_{k}\left(x_{1} \ldots x_{n}\right)^{\omega}$, where $y_{k} \neq x_{n}$ and $x_{1} \ldots x_{n}$ is not periodic, i.e., is not a proper power (note that every pre-periodic sequence can be uniquely represented in that form). Then we denote by $\Re(w)$ the group $\Omega\left(x_{1} \ldots x_{n-1}\right)$ in the first case and $\mathfrak{R}\left(y_{1} \ldots y_{k}, x_{1} \ldots x_{n}\right)$ in the second.

Theorem 5.1. Let $f(z)=z^{2}+c$ be a post-critically finite quadratic polynomial. Let $\theta \in \mathbb{R} / \mathbb{Z}$ be an angle such that the parameter ray $\mathbf{R}_{\theta}$ lands either on the root of the hyperbolic component $M_{c}$ (if $c$ is periodic) or on $c$ (if $c$ is pre-periodic).

Then $\operatorname{IMG}\left(z^{2}+c\right)$ is isomorphic to $\Omega(\hat{\theta})$. Moreover, the action of $\operatorname{IMG}\left(z^{2}+c\right)$ on the tree of preimages is conjugate with the action of $\Omega(\hat{\theta})$ on the binary tree. 
We consider independently the periodic and pre-periodic cases.

Proof in the periodic case. We use the invariant spiders, described in [14], to make cuts in $\overline{\mathbb{C}}$. A spider is a collection of disjoint closed paths $\gamma_{z}$, called legs, connecting every point $z \in P_{f}$ to infinity. A spider $\delta$ is $f$-invariant if $f^{-1}(\&) \supset \delta$, up to an isotopy relative to $P_{f}$.

The dynamical ray $\mathbf{R}_{\theta}$ has two preimages under $f$ : the ray $\mathbf{R}_{\theta / 2}$ and $\mathbf{R}_{(1+\theta) / 2}$. These rays land on two points belonging to the boundary of the Fatou component containing 0 . These two points are the preimages of the root of the component of $c$. Let us connect zero to these two points by internal rays of the Fatou component, i.e., by images of rays under the holomorphic map fixing 0 and mapping the component to the disc $\mathbb{D}$.

The union of the rays $\mathbf{R}_{\theta / 2}, \mathbf{R}_{(1+\theta) / 2}$ and the internal rays just constructed also divide the plane into two connected components. Let us denote the component to which $c$ belongs by $S_{1}$, and the other component (to which the ray $\mathbf{R}_{0}$ belongs) by $S_{0}$.

For each $i \in\{0,1\}$, the restriction of $z^{2}+c$ to the component $S_{i}$ is a homeomorphism of $S_{i}$ onto the set $\mathbb{C} \backslash \gamma_{c}$, where $\gamma_{c}$ is a curve connecting $c$ to infinity. The curve $\gamma_{c}$ is the union of the dynamical ray $\mathbf{R}_{\theta}$ with an internal ray.

Let $t \notin \bigcup_{n \geq 0} f^{k}\left(\gamma_{c}\right)$ be a basepoint and let $T=\bigsqcup_{n \geq 0} f^{-n}(t)$ be the corresponding tree of preimages. We will construct an isomorphism between the tree $T$ and the binary tree $\{0,1\}^{*}$ using itineraries.

If $z \in f^{-n}(t)$ is a vertex of the tree $T$, then the corresponding vertex of the binary tree is given by the word $\Lambda(z)=x_{n-1} x_{n-2} \ldots x_{0}$, where $x_{k} \in\{0,1\}$ is such that

$$
f^{k}(z) \in S_{x_{k}}
$$

It follows directly from the definition that $\Lambda$ is a level-preserving bijection and that if $\Lambda(z)=x_{n-1} \ldots x_{0}$, then $\Lambda(f(z))=x_{n-1} \ldots x_{1}$. Hence $\Lambda: T \rightarrow\{0,1\}^{*}$ is an isomorphism of rooted trees. From now on, we identify the trees $X^{*}$ and $T$ by this isomorphism.

Let $P_{f}=\bigcup_{i \geq 1} f^{r}(c)$ be the post-critical orbit of $f$, and set $\mathcal{M}=\mathbb{C} \backslash P_{f}$, so that $f$ is a covering map from $f^{-1}(\mathcal{M})$ to $\mathcal{M}$. If $n$ is the period of the point $c$, then $n$ is also the period of the curve $\gamma_{c}$ under iteration of $f$. Moreover, the curves $f^{k}\left(\gamma_{c}\right)$ are pairwise disjoint for $k=0,1, \ldots, n-1$. More precisely, they can have common points, but they do not intersect transversally, so that they become pairwise disjoint after small homotopies in $\mathcal{M}$, see [14]. Then the set $\left\{f^{k}\left(\gamma_{c}\right) \mid 0 \leq k \leq n-1\right\}$ is an invariant spider. For every $z \in P_{f}$ we denote by $\gamma_{z}$ the unique leg $f^{k}\left(\gamma_{c}\right)$ of the spider which connects the point $z$ to infinity. We have then $f\left(\gamma_{z}\right)=\gamma_{f(z)}$. The right-hand side of Figure 3 shows the preimage of a spider.

An example of spider $\bigcup_{z \in P_{f}} \gamma_{z}$ is shown on the left hand side of Figure 3, for $c \approx-0.1225+0.7448 i$ (the "Douady rabbit"). The corresponding $\theta$ is equal either to $1 / 7$ or to $2 / 7$ (it is $2 / 7$ in our picture), the critical point belongs to a cycle of length 3 and the root of all three components is a common fixed point. 

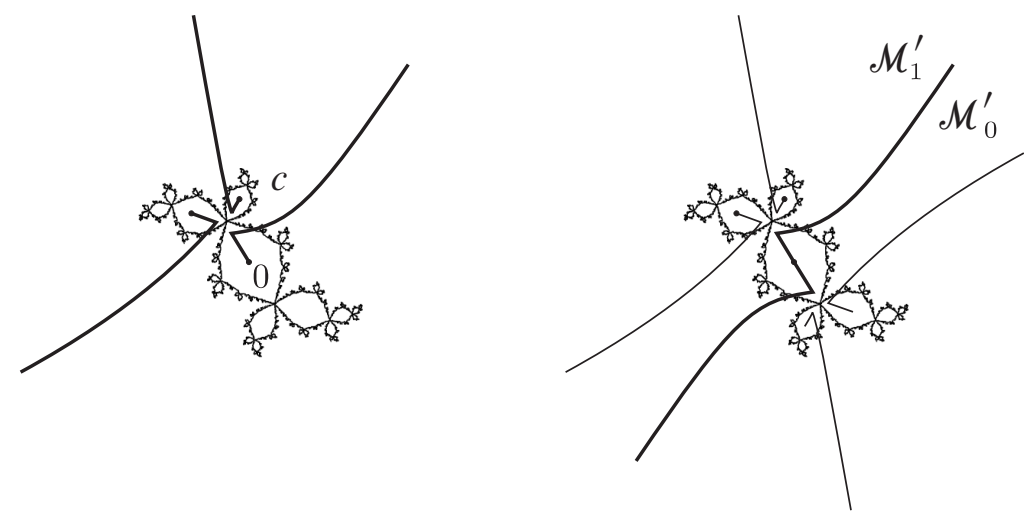

Figure 3. Invariant spider.

Write $\mathcal{M}^{\prime}=\mathcal{M} \backslash \bigcup_{z \in P_{f}} \gamma_{z}$. It follows that $\mathcal{M}^{\prime}$ is simply connected and $f^{-1}\left(\mathcal{M}^{\prime}\right) \subset$ $\mathcal{M}^{\prime}$, up to isotopy. The set $f^{-1}\left(\mathcal{M}^{\prime}\right)$ has two connected components $\mathcal{M}_{0}^{\prime}=S_{0} \cap \mathcal{M}^{\prime}$ and $\mathcal{M}_{1}^{\prime}=S_{1} \cap \mathcal{M}^{\prime}$.

Define for $t, u \in \mathcal{M}^{\prime}$ and $z \in P_{f}$ the path $g_{z}(t, u)$ starting in $t$, ending in $u$, disjoint from all the paths $\gamma_{y}$ of the spider for $y \neq z$, and intersecting $\gamma_{z}$ only once in such a direction that the part of $\gamma_{z}$ containing $z$ is to the left of the path $g_{z}(t, u)$. The path $g_{z}(t, u)$ is obviously defined uniquely up to homotopy in $\mathcal{M}$. We also denote by $g(t, u)$ the path starting in $t$, ending in $u$ and disjoint from the legs of the spider. The path $g(t, u)$ is also uniquely defined up to homotopy in $\mathcal{M}$. If $y \notin P_{f}$ then $g_{y}(t, u)$ is, by definition, the path $g(t, u)$.

It follows from the definitions that, for $z \neq c$,

$$
f^{-1}\left(g_{z}(t, u)\right)=\left\{g_{z_{0}}\left(t_{0}, u_{0}\right), g_{z_{1}}\left(t_{1}, u_{1}\right)\right\},
$$

where $f^{-1}(z)=\left\{z_{0}, z_{1}\right\}$ and $f^{-1}(t)=\left\{t_{0}, t_{1}\right\}$ and $f^{-1}(u)=\left\{u_{0}, u_{1}\right\}$. We may assume, up to changing our notation, that $z_{0} \in S_{0}$ and $z_{1} \in S_{1}$. Then $t_{0}, u_{0} \in S_{0}$ and $t_{1}, u_{1} \in S_{1}$.

If $z=c$, then the only preimage of $z$ is 0 and only one of the components of $f^{-1}\left(g_{c}(t, u)\right)$ intersects the path $\gamma_{0}$. Then this component is homotopic to $g_{0}\left(t_{0}, u_{0}\right)$, and the other component is homotopic to $g\left(t_{1}, u_{1}\right)$. If $\gamma_{0}$ is the extension of the ray $\mathbf{R}_{\theta / 2}$, then $t_{0}, u_{1} \in S_{0}$ and $t_{1}, u_{0} \in S_{1}$. If $\gamma_{0}$ is the extension of the ray $\mathbf{R}_{(1+\theta) / 2}$, then $t_{0}, u_{1} \in S_{1}$ and $t_{1}, u_{0} \in S_{0}$.

We also have that the $f$-preimages of the path $g(t, u)$ are the paths $g\left(t_{0}, u_{0}\right)$ and $g\left(t_{1}, u_{1}\right)$, where $t_{0}, u_{0} \in S_{0}$ and $t_{1}, u_{1} \in S_{1}$.

It is not hard to see now that if $\gamma_{0}$ is the extension of the ray $\mathbf{R}_{(1+\theta) / 2}$, then the generators $h_{k}=g_{f^{k}(0)}(t, t)$ of $\pi_{1}(\mathcal{M}, t)$ act on the tree $X^{*}$ in the same way as the 
generators $a_{k}$ of the group $\Omega(\hat{\theta})$. If $\gamma_{0}$ is the extension of the ray $\mathbf{R}_{\theta / 2}$, then the generators $h_{k}$ act in the same way as the generators $a_{k}^{-1}$.

We conclude that the actions of $\operatorname{IMG}(f)$ on $T$ and of $\Omega(\hat{f})$ on $X^{*}$ are conjugate. Note that the proof does not depend on the choice of $\theta$ for a given $c$ (though the curve $\gamma_{0}$ does depend on $\theta$ ).

The wreath recursion defining $\Omega(\hat{\theta})$ is given by the paths disjoint with the legs of the spider connecting the basepoint to its preimages. A different choice of the connecting paths (and hence of the wreath recursion) are convenient in some other situations (see, for instance [6]).

Proof in the pre-periodic case. As before, we consider dynamical rays $R_{\alpha}$. In the pre-periodic case, both preimages of $R_{\theta}$ land on 0 , and they divide the plane into two connected components. The problem here is that there is no invariant spider with disjoint legs, when the period of the angle is greater than that of the kneading sequence.

However, we can find a sequence of spiders $\delta_{0}, \delta_{1}, \ldots$, such that $\delta_{k+1} \subset$ $f^{-1}\left(\delta_{k}\right)$. Take any spider $\delta_{0}$ consisting of dynamical rays landing on $P_{f}$ and define inductively $\delta_{k}$ to be the set of paths belonging to $f^{-1}\left(\delta_{k-1}\right)$ and landing on the points of $P_{f}$. The points of $P_{f}$ are not critical, therefore for every $z \in P_{f}$ there exists a unique path $\gamma_{z, k+1} \in f^{-1}\left(\delta_{k-1}\right)$ landing on $z$. Hence the spider $\delta_{k}$ is well defined.

We can then define the paths $g_{z, k}(t, u)$ for every spider $\delta_{k}$ in the same way as in the periodic case. If $\gamma_{c, k}$ is the element of $\delta_{k}$ landing on $c$, then we denote by $S_{0, k+1}$ and $S_{1, k+1}$ the components of $\mathbb{C} \backslash f^{-1}\left(\gamma_{c, k}\right)$, where $S_{1, k+1}$ is the component containing $c$. The isomorphism $\Lambda: T \rightarrow X^{*}$ is then defined using the itineraries of points with respect to these partitions of the plane. Namely, if $z \in f^{-k}(t)$ is a vertex of the tree $T$, then $\Lambda(z)=x_{n-1} x_{n-2} \ldots x_{0}$, where $x_{k} \in\{0,1\}$ is such that

$$
f^{k}(z) \in S_{x_{k}, n-k} \text {. }
$$

It is also easy to prove that $\Lambda$ is an isomorphism of rooted trees.

The same formulae for the preimages of the paths $g_{z, k}(t, u)$ hold as in the periodic case. The only differences will be that the index $k+1$ appears at the preimages of paths and at names of the components $S_{i, k}$, and that the point 0 does not belong to the post-critical set. Therefore the preimages of $g_{c}(t, u)$ are the paths $g\left(t_{0}, u_{0}\right)$ and $g\left(t_{1}, u_{1}\right)$, with $t_{0}, u_{1} \in S_{0, k+1}$ and $t_{1}, u_{0} \in S_{1, k+1}$. The partitions of the plane into components $S_{0, k+1}$ and $S_{1, k+1}$ agree with the kneading sequence (i.e., every $z \in P_{f}$ belongs either only to the sectors $S_{0, k}$ or only to the sectors $S_{1, k}$ ), since the kneading sequences of all rays landing on $c$ are equal to $\hat{\theta}$. These considerations prove that the generators $g_{z, 0}(t, t)$ of $\operatorname{IMG}(f)$ act in the same way as the generators $a_{i}, b_{i}$ of the $\operatorname{group} \Omega(\hat{\theta})$. 
The following result follows from the classical results in symbolic dynamics of quadratic polynomials (see [9], [16]). It follows also from general results on iterated monodromy groups of expanding maps (see [17], Theorem 6.4.4).

Theorem 5.2. Under the conditions of Theorem 5.1, the limit dynamical system of the group $\Omega(\hat{\theta})$ is topologically conjugate to the action of the polynomial $z^{2}+c$ on its Julia set.

\section{References}

[1] L. Bartholdi, Endomorphic presentations of branch groups. J. Algebra 268 (2003), 419-443. Zbl 1044.20015 MR 2009317

[2] L. Bartholdi and R. I. Grigorchuk, Lie methods in growth of groups and groups of finite width. In Computational and geometric aspects of modern algebra (Edinburgh, 1998), London Math. Soc. Lecture Note Ser. 275, Cambridge University Press, Cambridge 2000, 1-27. Zbl 1032.20026 MR 1776763

[3] L. Bartholdi and R. I. Grigorchuk, On parabolic subgroups and Hecke algebras of some fractal groups. Serdica Math. J. 28 (2002), 47-90. Zbl 1011.20028 MR 1899368

[4] L. Bartholdi, R. I. Grigorchuk, and Z. Šunik, Branch groups. In Handbook of algebra. Vol. 3, North-Holland, Amsterdam 2003, 989-1112. Zbl 02156384 MR 2035113

[5] L. Bartholdi, V. A. Kaimanovich, and V. V. Nekrashevych, On amenability of automata groups. Preprint 2008. arXiv:0802.2837

[6] L. Bartholdi and V. Nekrashevych, Thurston equivalence of topological polynomials. Acta Math. 197 (2006), 1-51. Zbl 05114940 MR 2285317

[7] L. Bartholdi and B. Virág, Amenability via random walks. Duke Math. J. 130 (2005), 39-56. Zbl 1104.43002 MR 2176547

[8] E. Bondarenko and V. Nekrashevych, Post-critically finite self-similar groups. Algebra Discrete Math. 2003 (2003), no. 4, 21-32. Zbl 1068.20028 MR 2070400

[9] H. Bruin and D. Schleicher, Symbolic dynamics of quadratic polynomials. Report No. 7, 2001/2002, Institut Mittag-Leffler, The Royal Swedish Academy of Sciences. http://www.mittag-leffler.se/preprints/meta/BruinThu_Jun_13_13_59_29.rdf.html

[10] A. Douady and J. H. Hubbard, Étude dynamique des polynômes complexes (Première partie). Publ. Math. Orsay 2, Université de Paris-Sud, Paris 1984. Zbl 0552.30018 MR 0762431

[11] A. Douady and J. H. Hubbard, Étude dynamique des polynômes complexes (Deuxième partie). Publ. Math. Orsay 4, Université de Paris-Sud, Paris 1985. Zbl 0571.30026 MR 0812271

[12] A. Erschler, Boundary behavior for groups of subexponential growth. Ann. of Math. (2) 160 (2004), 1183-1210. Zbl 1089.20025 MR 2144977 
[13] R. I. Grigorchuk, Burnside's problem on periodic groups. Funktsional. Anal. i Prilozhen. 14 (1980), 53-54; English transl. Funct. Anal. Appl. 14 (1980), 41-43. Zbl 0595.20029 MR 565099

[14] J. H. Hubbard and D. Schleicher, The spider algorithm. In Complex dynamical systems (Cincinnati, OH, 1994), Proc. Sympos. Appl. Math. 49, Amer. Math. Soc., Providence, RI, 1994, 155-180. Zbl 0853.58088 MR 1315537

[15] L. Kaloujnine and M. Krasner, Le produit complet des groupes de permutations et le problème d'extension des groupes. C. R. Acad. Sci. Paris 227 (1948), 806-808. Zbl 0038.16203 MR 0027758

[16] K. Keller, Invariant factors, Julia equivalences and the (abstract) Mandelbrot set. Lecture Notes in Math. 1732, Springer-Verlag, Berlin 2000. Zbl 0970.37032 MR 1761576

[17] V. Nekrashevych, Self-similar groups. Math. Surveys Monogr. 117, Amer. Math. Soc., Providence, RI, 2005. Zbl 1087.20032 MR 2162164

[18] S. Sidki, Automorphisms of one-rooted trees: growth, circuit structure, and acyclicity. J. Math. Sci. 100 (2000), 1925-1943. Zbl 1069.20504 MR 1774362

Received December 16, 2006; revised February 19, 2008

L. Bartholdi, Institut de Mathématiques B (IMB), École Polytechnique Fédérale de Lausanne (EPFL), 1015 Lausanne, Switzerland

E-mail: laurent.bartholdi@gmail.com

V.V. Nekrashevych, Department of Mathematics, Texas A\&M University, College Station, TX 77843-3368, U.S.A.

E-mail: nekrash@math.tamu.edu 